\title{
CANONICAL INTEGRAL STRUCTURES ON THE DE RHAM COHOMOLOGY OF CURVES
}

\author{
BRYDEN CAIS
}

\begin{abstract}
For a smooth and proper curve $X_{K}$ over the fraction field $K$ of a discrete valuation ring $R$, we explain (under very mild hypotheses) how to equip the de Rham cohomology $H_{\mathrm{dR}}^{1}\left(X_{K} / K\right)$ with a canonical integral structure: i.e. an $R$-lattice which is functorial in finite (generically étale) $K$-morphisms of $X_{K}$ and which is preserved by the cup-product auto-duality on $H_{\mathrm{dR}}^{1}\left(X_{K} / K\right)$. Our construction of this lattice uses a certain class of normal proper models of $X_{K}$ and relative dualizing sheaves. We show that our lattice naturally contains the lattice furnished by the (truncated) de Rham complex of a regular proper $R$-model of $X_{K}$ and that the index for this inclusion of lattices is a numerical invariant of $X_{K}$ (we call it the de Rham conductor). Using work of Bloch and of Liu-Saito, we prove that the de Rham conductor of $X_{K}$ is bounded above by the Artin conductor, and bounded below by the Efficient conductor. We then study how the position of our canonical lattice inside the de Rham cohomology of $X_{K}$ is affected by finite extension of scalars.
\end{abstract}

\section{INTRODUCTION}

Let $R$ be a discrete valuation ring with field of fractions $K$ and residue field $k$, and consider a smooth proper (not necessarily connected) curve $X_{K}$ over $K$. The Hodge to de Rham spectral sequence degenerates [13], so there is a short exact sequence of finite dimensional $K$-vector spaces

$$
0 \longrightarrow H^{0}\left(X_{K}, \Omega_{X_{K} / K}^{1}\right) \longrightarrow H_{\mathrm{dR}}^{1}\left(X_{K} / K\right) \longrightarrow H^{1}\left(X_{K}, \mathscr{O}_{X_{K}}\right) \longrightarrow 0 .
$$

This exact sequence is auto-dual with respect to the cup-product pairing, and is both contravariantly and covariantly functorial (via pullback and trace) in finite generically étale ${ }^{1}$ morphisms of smooth curves, so in particular is equipped with an action of the $\mathbf{Z}$-algebra of correspondences on $X_{K}$.

By an integral structure on (1.1), we mean a short exact sequence of free $R$-modules that recovers (1.1) after extending scalars to $K$. The main aim of this paper is to equip (1.1) with a canonical integral structure, i.e. one that is functorial in both pullback and trace by finite generically étale $K$ morphisms of $X_{K}$, hence stable by the action of correspondences, and which is self-dual with respect to the cup-product pairing.

Our main result is Theorem 5.11, which states that when $X_{K}$ has an admissible model over $R$ (see Definition 2.4), then there is a canonical integral structure on (1.1). We remark that $X_{K}$ has such a model when, for example, each connected component has a $K^{\prime}$-rational point for some tamely ramified extension $K^{\prime}$ of $K$ (so a fortiori when $X_{K}$ has good or semistable reduction), or more generally when $X_{K}$ has a regular model which is cohomologically flat (see Remark 3.2 and Proposition 3.3). In

2000 Mathematics Subject Classification. Primary: 14F40; Secondary: 11G20, 14F30, 14G20, $14 \mathrm{H} 25$.

Key words and phrases. de Rham cohomology, p-adic local Langlands, curve, rational singularities, arithmetic surface, Grothendieck duality, Artin conductor, Efficient conductor, simultaneous resolution of singularities.

This work was partially supported by the NSF grant DMS-0502170 and by a Rackham Predoctoral Fellowship.

${ }^{1}$ Note that generic étaleness is needed in order to have a trace morphism on functions. 
particular, our theorem holds for modular curves over $p$-adic fields with arbitrary $p^{n}$-level, which in general can be very far from having semistable reduction; see Remark 2.11. Furthermore, we point out that the formation of our canonical integral structure on (1.1) commutes with base change to the closed fiber; i.e. the reduction of our integral structure modulo a uniformizer of $R$ can be described in terms of the geometry of the closed fiber of any admissible model $X$ of $X_{K}$.

Let us briefly explain our motivation for studying canonical integral structures in the de Rham cohomology of curves. Consider the tower of modular curves $\left\{X\left(\Gamma_{r}\right)\right\}_{r}$ over $\mathbf{Q}_{p}$ where $\Gamma_{r}$ is the congruence subgroup $\Gamma\left(p^{r}\right) \cap \Gamma_{1}(N)$ for some fixed integer $N$ coprime to $p$. For each $r$, the $p$-adic étale cohomology groups $H_{\text {ét }}^{1}\left(X\left(\Gamma_{r}\right)_{\overline{\mathbf{Q}}_{p}}, \mathbf{Z}_{p}\right)$ are finite $\mathbf{Z}_{p}$-modules equipped with a natural action of $G_{\mathbf{Q}_{p}}:=\operatorname{Gal}\left(\overline{\mathbf{Q}}_{p} / \mathbf{Q}_{p}\right)$. Since the "forget the $p^{r}$-level structure" map $X\left(\Gamma_{r}\right) \rightarrow X\left(\Gamma_{0}\right)$ is Galois with Galois group $\mathrm{GL}_{2}\left(\mathbf{Z} / p^{r} \mathbf{Z}\right)$, one obtains a $p$-adic Banach space representation of $\mathrm{GL}_{2}\left(\mathbf{Q}_{p}\right)$

$$
\widetilde{H}_{\mathbf{Q}_{p}}^{1}:=(\underbrace{\lim }_{s} \underset{r}{\lim } H_{\text {ét }}^{1}\left(X\left(\Gamma_{r}\right)_{\overline{\mathbf{Q}}_{p}}, \mathbf{Z}_{p}\right) /\left(p^{s}\right)) \otimes \mathbf{z}_{p} \mathbf{Q}_{p}
$$

that is moreover equipped with an action of $G_{\mathbf{Q}_{p}}$. In [17], Emerton shows that $\widetilde{H}_{\mathbf{Q}_{p}}^{1}$ is an admissible representation of $\mathrm{GL}_{2}\left(\mathbf{Q}_{p}\right)$ (in the sense of Schneider-Teitlebaum [33]) which encodes many deep properties about $p$-adic families of modular forms and their Galois representations. Note that for each $r$, the (Galois) representations $H_{\text {ett }}^{1}\left(X\left(\Gamma_{r}\right), \mathbf{Q}_{p}\right)$ are de Rham, so $p$-adic Hodge theory provides a natural isomorphism of filtered $\mathrm{GL}_{2}\left(\mathbf{Z} / p^{r} \mathbf{Z}\right)$-representations

$$
\left(H_{\text {ét }}^{1}\left(X\left(\Gamma_{r}\right), \mathbf{Q}_{p}\right) \otimes \mathbf{Q}_{p} B_{\mathrm{dR}}\right)^{G_{\mathbf{Q}_{p}}} \simeq H_{\mathrm{dR}}^{1}\left(X\left(\Gamma_{r}\right) / \mathbf{Q}_{p}\right)
$$

With (1.2) in mind, it is natural to ask if one can define a de Rham analogue of $\widetilde{H}_{\mathbf{Q}_{p}}^{1}$. This analogue should be an admissible ( $p$-adic Banach) $\mathrm{GL}_{2}\left(\mathbf{Q}_{p}\right)$-representation equipped with a decreasing filtration by admissible $\mathrm{GL}_{2}\left(\mathbf{Q}_{p}\right)$ sub-representations. In order to $p$-adically complete, one needs an integral version of $H_{\mathrm{dR}}^{1}\left(X\left(\Gamma_{r}\right) / \mathbf{Q}_{p}\right)$ and its Hodge filtration that is "sufficiently functorial" so as to be equipped with a $\mathrm{GL}_{2}\left(\mathbf{Z} / p^{r} \mathbf{Z}\right)$-action. For large $r$, the Galois representations $H_{\text {ét }}^{1}\left(X\left(\Gamma_{r}\right), \mathbf{Q}_{p}\right)$ are very far from being semistable in the sense that they become semistable only after a large, wildly ramified extension of $\mathbf{Q}_{p}$. Thus, the issue of an appropriate integral structure on $H_{\mathrm{dR}}^{1}\left(X\left(\Gamma_{r}\right) / \mathbf{Q}_{p}\right)$ is a subtle one; in particular, integral de Rham cohomology seems to be the "wrong" lattice (i.e. integral structure) to use, for a variety of reasons. Our lattices have three favorable features: they are stable under the action of correspondences on the curve, they are auto-dual with respect to cup-product, and their reductions modulo $p$ can be described in terms of the geometry of the closed fiber of any admissible model of $X$. For these reasons, it is our hope that the canonical integral structures studied in this paper will enable us to construct the right de Rham analogue of $\widetilde{H}_{\mathbf{Q}_{p}}^{1}$, and that some "completed" analogue of the $p$-adic Hodge theory isomorphism (1.2) will hold.

There are essentially two ways to construct a canonical integral structure on (1.1). One method proceeds via the Néron model of the Jacobian of $X_{K}$ and the theory of canonical extensions of Néron models (see [29, I, 55]). The other method employs a careful study of a certain class of models of $X_{K}$, and works directly with the geometry of arithmetic surfaces. We take the second approach in this paper, which has the advantage that many aspects of the lattices we obtain seem simpler to analyze via models of curves than via Néron models of Jacobians; in particular, we can prove quite a bit more via this method. We refer the reader to [8] for the first approach, and note that when $X_{K}$ has a 
model over $R$ with generically smooth closed fiber, the two methods yield lattices which are naturally isomorphic (see [8]).

We now explain the main idea of our construction. When $X_{K}$ has good reduction over $R$, a simple argument with minimal regular models shows that the Hodge filtration of the de Rham cohomology of a smooth proper model over $R$ yields a canonical integral structure on (1.1). When $X_{K}$ does not have good reduction, the (relative) de Rham cohomology of a proper and flat (even regular!) model $X$ can in general be rather pathological. Roughly, there are two problems: first, the sheaf of relative differentials of a proper flat regular model $X$ is not generally a flat $\mathscr{O}_{X}$-module, resulting in various torsion phenomena in de Rham cohomology. Second, and perhaps more seriously, when $X$ is not $R$-smooth, the sheaf of relative differentials $\Omega_{X / R}^{1}$ is not the relative dualizing sheaf, so one can not expect integral de Rham cohomology to have a good duality theory (i.e. trace maps attached to finite morphisms of curves and cup-product auto-duality). To remedy the situation, for a proper flat and normal model $X$ of $X_{K}$ over $R$, we will study a certain sub-complex $d: \mathscr{O}_{X} \rightarrow \omega_{X / R}$ of the de Rham complex of $X_{K}$, where $\omega_{X / R}$ is the relative dualizing sheaf of $X$ over $R$. We will show that when $X$ is cohomologically flat over $R$, the hypercohomology of this complex provides an integral structure on (1.1). In general, this integral structure may depend on the choice of model $X$, but we will show that when $X$ is admissible (Definition 2.4), the resulting integral structure is independent of the choice of admissible model. Given such independence, proving that the resulting integral structure is functorial in finite $K$-morphisms of $X_{K}$ then comes down to the problem of showing that a finite morphism of curves over $K$ (that have admissible models over $R$ ) may be extended to a finite morphism of admissible models. This is established in Theorem 3.4 using the flattening techniques of Gruson-Raynaud [32]. We note that the corresponding extension property of finite morphisms is false if one insists on working with regular models [26], [1]; thus, it is essential that we do not require our admissible models to be regular (only normal).

The major technical tool in what we do is Grothendieck duality ([19], [9]). Thus, after a brief study of the geometry and properties of the class of admissible curves and models in $\S 2-\S 3$, for the convenience of the reader and for lack of an adequate reference in the literature, we work out in detail Grothendieck duality for relative curves in $\S 4$. The heart of this work is $\S 5$, where we study the twoterm complex $\mathscr{O}_{X} \rightarrow \omega_{X / R}$ and its hypercohomology. When $R$ is the localization at some finite place of the ring of integers in a number field and $X$ is regular, this hypercohomology occurs in Bloch's work [5, $\S 2]$ on the Artin conductor of $X / R$. In $\S 6$, assuming that $R$ has perfect residue field and that $X_{K}$ is geometrically connected, we use Bloch's results (and subsequent work of Liu-Saito [27]) to define a numerical invariant, the de Rham conductor of $X_{K} / K$ (Definition 6.6), which measures how far our lattice in $H_{\mathrm{dR}}^{1}\left(X_{K} / K\right)$ is from the lattice furnished by the image of integral de Rham cohomology, and we prove that this invariant is bounded above by the Artin conductor of $X_{K} / K$ (see Theorem 6.7). We conclude in $\S 7$ by addressing the following natural question: if $K^{\prime} / K$ is any finite separable extension, then what is the relationship between the canonical integral structure on $H_{\mathrm{dR}}^{1}\left(X_{K^{\prime}} / K^{\prime}\right)$ and the lattice furnished (via extension of scalars) by the canonical integral structure on $H_{\mathrm{dR}}^{1}\left(X_{K} / K\right)$ ? When $K^{\prime}$ is an unramified extension of $K$, these two lattices coincide, while for general (ramified) extensions, they need not agree (nor is it the case that one lattice necessarily contains the other; see Remarks 7.2). Theorem 7.1 provides precise estimates for the relative positions of these two lattices inside $H_{\mathrm{dR}}^{1}\left(X_{K^{\prime}} / K^{\prime}\right)$ in general. 
Throughout, our hypotheses and methods are kept as general as possible, so that our results will apply to many curves of arithmetic interest. In particular, we do not require $K$ to have characteristic zero. Moreover, we wish to emphasize that our constructions work for quite general curves; in particular, we do not impose any semi-stability hypotheses. As we have indicated, one of the main applications of our theory that we have in mind is to the case of the modular curves $X\left(\Gamma_{r}\right)$ over $\mathbf{Q}_{p}$. These curves do not have geometrically connected generic fiber. Moreover, it is natural in proofs to have to extend the ground field, which may destroy connectivity, and so (with the exception of $\S 6$ where we need geometric connectivity) we do not assume that $X_{K}$ connected.

It is a pleasure to thank Spencer Bloch, Matthew Emerton, Dino Lorenzini, Bill Messing, Martin Olsson, Takeshi Saito, and Karen Smith for several helpful exchanges and conversations. I would especially like to thank Brian Conrad, both for advising the author's Ph.D thesis - which was the genesis of this manuscript - and for many clarifications and corrections on an earlier version. I heartily thank the referee for correcting a number of errors and for suggesting several improvements.

\section{Contents}

1. Introduction

2. Admissible curves

3. Admissible models

4. The dualizing sheaf and Grothendieck duality

5. Cohomology of regular differentials

6. Comparison with integral de Rham cohomology

7. Base change

References

\section{Admissible CuRves}

We keep the notation from the introduction, and we put $S=\operatorname{Spec} R$. In this section, we will introduce and study the class of models of $X_{K}$ over $S$ that we will use to equip the de Rham cohomology of $X_{K}$ with a canonical integral structure.

By a (relative) curve over $S$ we mean a flat, finite type, and separated $S$-scheme $f: X \rightarrow S$ of pure relative dimension 1 that is normal with smooth generic fiber. For technical convenience, we do not require relative curves to be proper or connected over $S$. Note that the non-regular locus of a curve is a finite subset of closed points of the closed fiber: when $R$ is excellent this is well-known [18, $I V_{2}$, 7.8.6 (iii)], and in general - since $X_{K}$ is smooth - it follows from the corresponding statement for the base change $X \otimes_{R} \widehat{R}$ to the excellent completion $\widehat{R}$ of $R$ by [11, Lemma 2.1.1] (note that this uses the fact that a local noetherian ring is regular if it admits a regular faithfully flat extension [28, Theorem 23.7])

In order to define the class of relative curves that we consider, we must first recall the definitions of cohomological flatness and of rational singularities.

Fix a proper relative curve $f: X \rightarrow S$. Recall that $f$ is said to be cohomologically flat (in dimension zero) if the formation of $f_{*} \mathscr{O}_{X}$ commutes with arbitrary base change. For our purposes, the significance of cohomological flatness is the following: 
Lemma 2.1. Let $f$ be as above, and let $\mathscr{F}$ be any $S$-flat coherent sheaf on $X$. Then the formation of $f_{*} \mathscr{F}$ commutes with arbitrary base change if and only if $H^{1}(X, \mathscr{F})$ is torsion-free; in particular, $f$ is cohomologically flat in dimension zero if and only if the $R$-module $H^{1}\left(X, \mathscr{O}_{X}\right)$ is torsion-free.

Proof. Let $i: X_{k} \hookrightarrow X$ be the inclusion of the closed fiber and let $\pi \in R$ be a uniformizer. Since $\mathscr{F}$ is $S$-flat, we have a short exact sequence

$$
0 \longrightarrow H^{0}(X, \mathscr{F}) \otimes_{R} k \longrightarrow H^{0}\left(X_{k}, i^{*} \mathscr{F}\right) \longrightarrow H^{1}(X, \mathscr{F})[\pi] \longrightarrow 0,
$$

where $H^{1}(X, \mathscr{F})[\pi]$ denotes the $\pi$-torsion submodule of $H^{1}(X, \mathscr{F})$. We conclude that the formation of $f_{*} \mathscr{F}$ commutes with the base change Spec $k \rightarrow S$ (and hence arbitrary base change) if and only if $H^{1}(X, \mathscr{F})$ is torsion-free.

By resolution of singularities for excellent surfaces [22] and descent arguments from $\widehat{R}$ (see [11, Theorem 2.2.2]) it follows that $X$ admits a resolution of singularities; i.e. there exists a proper birational morphism $\rho: X^{\prime} \rightarrow X$ with $X^{\prime}$ regular. $^{2}$ The higher direct image sheaf $R^{1} \rho_{*} \mathscr{O}_{X^{\prime}}$ is then supported at the finitely many non-regular points of $X$; we say that $X$ has rational singularities if there exists some such resolution with $R^{1} \rho_{*} \mathscr{O}_{X^{\prime}}=0$. Trivially, any regular $X$ has rational singularities. We will make frequent use of the following fact due to Lipman [21, Proposition 1.2, (2)], which shows in particular that whether or not $X$ has rational singularities may be checked using any resolution of singularities.

Proposition 2.2. The scheme $X$ has rational singularities if and only if for every proper birational morphism $\rho: X^{\prime} \rightarrow X$ with $X^{\prime}$ normal, we have $R^{1} \rho_{*} \mathscr{O}_{X^{\prime}}=0$.

Remark 2.3. Since we require $X$ to be normal, an equivalent formulation of rational singularities is that the natural derived category map $\mathscr{O}_{X} \rightarrow \mathbf{R} \rho_{*} \mathscr{O}_{X^{\prime}}$ is a quasi-isomorphism for every proper birational map $\rho: X^{\prime} \rightarrow X$ with $X^{\prime}$ normal.

We can now introduce our primary object of study in this section:

Definition 2.4. A proper relative curve $f: X \rightarrow S$ is admissible provided $f$ is cohomologically flat and $X$ has rational singularities.

Remark 2.5. Our definition of admissible generalizes that of Mazur and Ribet [30, $\S 2]$ in that we impose weaker conditions on the possible singularities of $X$ and we do not require the closed fiber to be reduced. Any proper semistable curve over $S$ is admissible, but the class of admissible curves is strictly larger than the class of proper semistable curves (see Example 2.10 and Remark 2.11).

We have formulated the notion of admissibility to ensure that every admissible curve over $S$ possesses certain properties. For example, in order for the $R$-module $H^{1}\left(X, \mathscr{O}_{X}\right)$ to yield a "good" integral structure on $H^{1}\left(X_{K}, \mathscr{O}_{X_{K}}\right)$, it is crucial that it be torsion free (such freeness will also be essential for later proofs to work). The hypothesis that $X$ has rational singularities is also critical for our purposes, as it enables us to blow up $X$ at closed points of the closed fiber (and then normalize) without changing the cohomology $H^{1}\left(X, \mathscr{O}_{X}\right)$ :

\footnotetext{
${ }^{2}$ This fact was first proved by Deligne and Mumford [14, 2$]$, using the same descent arguments from $\widehat{R}$ together with results of Abhyankar [2] and of Hironaka.
} 
Proposition 2.6. Let $f: X \rightarrow S$ be a proper relative curve with rational singularities and let $\rho: X^{\prime} \rightarrow$ $X$ be any proper birational morphism with $X^{\prime}$ normal. Then $X^{\prime}$ has rational singularities, and the canonical pullback map $H^{1}\left(X, \mathscr{O}_{X}\right) \rightarrow H^{1}\left(X^{\prime}, \mathscr{O}_{X^{\prime}}\right)$ is an isomorphism. In particular, $X^{\prime}$ is admissible if and only if $X$ is.

Proof. Since $X$ has rational singularities and $X^{\prime}$ is normal, Proposition 3.2 (ii) of [4] ensures ${ }^{3}$ that $X^{\prime}$ has rational singularities. Denoting by $f^{\prime}: X^{\prime} \rightarrow S$ the structural morphism, the exact sequence of terms of low degree of the spectral sequence $R^{m} f_{*} R^{n} \rho_{*} \mathscr{O}_{X^{\prime}} \Longrightarrow R^{m+n} f_{*}^{\prime} \mathscr{O}_{X^{\prime}}$ reads

$$
0 \longrightarrow R^{1} f_{*}\left(\rho_{*} \mathscr{O}_{X^{\prime}}\right) \longrightarrow R^{1} f_{*}^{\prime} \mathscr{O}_{X^{\prime}} \longrightarrow f_{*} R^{1} \rho_{*} \mathscr{O}_{X^{\prime}}
$$

By Proposition 2.2, we have $R^{1} \rho_{*} \mathscr{O}_{X^{\prime}}=0$. Thus, since the canonical map $\mathscr{O}_{X} \rightarrow \rho_{*} \mathscr{O}_{X^{\prime}}$ is an isomorphism (as $\rho$ is proper birational and $X$ is normal), we deduce that the pullback map $H^{1}\left(X, \mathscr{O}_{X}\right) \rightarrow$ $H^{1}\left(X^{\prime}, \mathscr{O}_{X^{\prime}}\right)$ is an isomorphism. It now follows from Lemma 2.1 that $X^{\prime}$ is admissible if and only if $X$ is.

As both cohomological flatness and rational singularities have somewhat abstract definitions, we wish to record some useful criteria that can be used in practice to show that many curves of arithmetic interest are admissible (see Example 2.10 and Remark 2.11).

Proposition 2.7 (Raynaud). Let $X$ be a connected proper relative $S$-curve. If char $(k)=0$ then $X$ is cohomologically flat. If char $(k)=p>0$ then $X$ is cohomologically flat if the greatest common divisor of the geometric multiplicities of the components of the closed fiber is prime to $p$, or a fortiori if $X$ admits an étale quasi-section.

Proof. By standard arguments (see, for example, the proof of Corollary 9.1.24 in [24]), we may reduce to the case that $X_{K}$ is geometrically connected. With this assumption we have $f_{*} \mathscr{O}_{X} \simeq \mathscr{O}_{S}$, so since $X$ is normal it follows $[31,6.1 .6]$ that $X$ satisfies the hypotheses of Raynaud's "critère de platitude cohomologique" [31, Théorème 7.2.1], which immediately implies the proposition.

Proposition 2.8. Let $f: X^{\prime} \rightarrow X$ be a finite generically étale surjective map of 2-dimensional excellent schemes that are normal and integral, and assume that $X^{\prime}$ has rational singularities. If $\rho: Y \rightarrow X$ is a resolution of singularities, then $\operatorname{deg} f$ annihilates $R^{1} \rho_{*} \mathscr{O}_{Y}$. In particular, if $\operatorname{deg} f$ is a unit in $\mathscr{O}_{X}$ then $R^{1} \rho_{*} \mathscr{O}_{Y}=0$

Note that a resolution of singularities $\rho: Y \rightarrow X$ always exists thanks to Lipman [22].

Proof. Let $g: Y^{\prime} \rightarrow Y$ be the normalization of $Y$ in the function field of $X^{\prime}$. As $f$ is generically étale, the function field of $X^{\prime}$ is a separable extension of the function field of $X$, so the map $g$ is finite. Setting $n:=\operatorname{deg} g=\operatorname{deg} f$ (generic degree), we thus have a commutative diagram

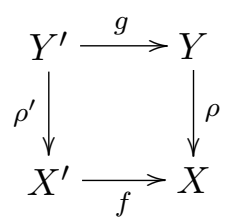

of normal 2-dimensional noetherian excellent schemes with $g$ and $f$ finite of generic degree $n$.

\footnotetext{
${ }^{3}$ Note that the proof given there does not require $R$ to be excellent.
} 
Since $Y$ is regular and $Y^{\prime}$ is normal (hence Cohen-Macaulay, as $Y^{\prime}$ has dimension 2) and the map $g$ is finite, we conclude by [28, Theorem 23.1] that $g$ is flat. Thus, $g_{*} \mathscr{O}_{Y^{\prime}}$ is finite locally free over $\mathscr{O}_{Y}$, and we have a (ring-theoretic) trace map

$$
\operatorname{tr}_{g}: g_{*} \mathscr{O}_{Y^{\prime}} \rightarrow \mathscr{O}_{Y}
$$

with the property that the composite map

$$
\mathscr{O}_{Y} \stackrel{g^{*}}{\longrightarrow} g_{*} \mathscr{O}_{Y^{\prime}} \stackrel{\operatorname{tr}_{g}}{\longrightarrow} \mathscr{O}_{Y}
$$

is multiplication by $n$.

We have quasi-isomorphisms

$$
\mathbf{R} \rho_{*} \mathbf{R} g_{*} \mathscr{O}_{Y^{\prime}} \simeq \mathbf{R}(\rho \circ g)_{*} \mathscr{O}_{Y^{\prime}}=\mathbf{R}\left(f \circ \rho^{\prime}\right)_{*} \mathscr{O}_{Y^{\prime}} \simeq \mathbf{R} f_{*} \mathbf{R} \rho_{*}^{\prime} \mathscr{O}_{Y^{\prime}} \simeq f_{*} \rho_{*}^{\prime} \mathscr{O}_{Y^{\prime}}
$$

where the last quasi-isomorphism results because $f$ is finite and $X^{\prime}$ has rational singularities (see Remark 2.3). Thus, $\mathbf{R} \rho_{*} \mathbf{R} g_{*} \mathscr{O}_{Y^{\prime}} \simeq \rho_{*} g_{*} \mathscr{O}_{Y^{\prime}}$, so by applying the functor $\mathbf{R} \rho_{*}$ to the composite map (2.1) and recalling that $g$ is finite ( $\operatorname{so} g_{*} \mathscr{O}_{Y^{\prime}} \simeq \mathbf{R} g_{*} \mathscr{O}_{Y^{\prime}}$ ) we deduce that multiplication by $n$ on $\mathbf{R} \rho_{*} \mathscr{O}_{Y^{\prime}}$ factors through $\rho_{*} g_{*} \mathscr{O}_{Y^{\prime}}$. Applying the functor $H^{1}$ on complexes gives the result.

The following corollary is usually attributed to Elkik [16, II, Lemme 1]:

Corollary 2.9. Let $A$ be an excellent 2-dimensional regular domain that is essentially of finite type over a noetherian domain $R$, and let $G$ be a finite group acting on $A$ by $R$-algebra automorphisms. If $\# G$ is a unit in $A$ then the ring of $G$-invariants $A^{G}$ has rational singularities.

Proof. It is easy to see that $A^{G}$ is normal, and our assumptions ensure that $A^{G} \rightarrow A$ is finite. Thus, the corollary follows at once from Proposition 2.8 with $X=\operatorname{Spec}\left(A^{G}\right)$ and $X^{\prime}=\operatorname{Spec} A$, using Artin's lemma to ensure that $\operatorname{Frac}(A)^{G}=\operatorname{Frac}\left(A^{G}\right) \rightarrow \operatorname{Frac}(A)$ is finite Galois of degree dividing \#G.

Example 2.10. Using Corollary 2.9, we can show that many relative $S$-curves of arithmetic interest have rational singularities. Quite generally, let $\mathscr{X}$ be any regular separated Deligne-Mumford stack of finite type and relative dimension one over $S$ and suppose that the finite order of the automorphism group at each geometric point of $\mathscr{X}$ is a unit in $R$. If the underlying coarse moduli space $X$ is a scheme, then it is a relative curve over $S$ with all strictly henselian local rings of the form $A^{G}$ with $A$ regular and $G$ a finite group of $R$-linear automorphisms of $A$ with order a unit in $R$. Since whether or not a local ring has a rational singularity can be checked on a strict henselization (as the formation of a resolution commutes with ind-étale base change), it follows that in this situation $X$ has rational singularities.

Remark 2.11. Consider the modular curve $X(N)_{\mathbf{Q}}$ over $\mathbf{Q}$. It is smooth and proper, and is the $\mathbf{Q}$-fiber of the coarse moduli space $X(N)$ associated to a certain regular Deligne-Mumford stack $\mathscr{M}_{\Gamma(N)}$ that is proper and flat over $\mathbf{Z}$ [10]. Since the automorphism group of any geometric point must divide 24 (as the automorphism group of an elliptic curve has order dividing 24 and the automorphism groups at cuspidal geometric points have order dividing 2) we conclude from the example above that $X(N) \mathbf{z}_{(p)}$ has rational singularities for $p>3$. Furthermore, the cusp $\infty$ is a Q-rational point of $X(N)_{\mathbf{Q}}$, so by Proposition 2.7 we see that $X(N)_{\mathbf{Q}}$ has an admissible model over $\mathbf{Z}_{(p)}$ for $p>3$.

Similar reasoning shows that the modular curve $X_{0}(N)_{\mathbf{Q}}$ has an admissible model over $\mathbf{Z}_{(p)}$ when $p>3$ (see Proposition 3 of $[7, \S 9.7]$ ). 
Remark 2.12. For a proper relative $S$-curve $X$, both the properties of "being cohomologically flat" and of "having rational singularities" have nice interpretations in terms of the relative Picard functor Pic $_{X / S}$ of $X$ over $S$. On the one hand, thanks to Artin [3, Theorem 7.3] and Raynaud [31, Proposition 5.2], the functor $\operatorname{Pic}_{X / S}$ is an algebraic space if and only if $f$ is cohomologically flat. On the other hand, let $\mathrm{Pic}_{X / S}^{0}$ be the identity component (see [7, pg. 154]) of the relative Picard functor and denote by $J^{0}$ the identity component of the Néron model of the Jacobian of $X_{K}$. Let us suppose that $\operatorname{Pic}_{X / S}^{0}$ is a scheme (which holds, for example, when the greatest common divisor of the geometric multiplicities of the components of the closed fiber of $X$ is 1 ; see $[7, \S 9.4$ Theorem 2 (b)]). Then the canonical map

$$
\operatorname{Pic}_{X / S}^{0} \longrightarrow J^{0}
$$

deduced (via the Néron mapping property) from the identification $\operatorname{Pic}_{X_{K} / K} \simeq J_{K}$ is an isomorphism if and only if $X$ has rational singularities. We will not need either of these characterizations in this paper (but they both play an essential role in the main comparison theorem of [8]).

\section{Admissible models}

Fix a smooth proper curve $X_{K}$ over $K$. By a model of $X_{K}$, we mean a proper curve (recall our conventions) $X$ over $S$ whose generic fiber is isomorphic to $X_{K}$ (although we will not explicitly keep track of the generic fiber identification). A model $X$ of $X_{K}$ is admissible if the relative curve $X$ is admissible in the sense of Definition 2.4. In this section, we study the admissible models of $X_{K}$.

Lemma 3.1. Any two admissible models can be dominated by a third.

Proof. First note that if $C$ is any flat, finite type, and separated $S$-scheme of pure relative dimension 1 having smooth generic fiber then the normalization map $\widetilde{C} \rightarrow C$ is finite. Indeed, by smoothness of $C_{K}$ we see that the base change of the normalization $(\widetilde{C})_{\widehat{R}} \rightarrow C_{\widehat{R}}$ is dominated by the normalization of the base change $\widetilde{\left(C_{\widehat{R}}\right)} \rightarrow C_{\widehat{R}}$, and the latter map is finite as $\widehat{R}$ is excellent.

If $X_{1}$ and $X_{2}$ are admissible models of $X_{K}$, we let $\Gamma_{K}$ denote the graph of the generic fiber isomorphism $\left(X_{1}\right)_{K} \simeq\left(X_{2}\right)_{K}$ in the fiber product $\left(X_{1}\right)_{K} \times_{K}\left(X_{2}\right)_{K}$. Let $\Gamma$ be the closure of $\Gamma_{K}$ in $X_{1} \times_{S} X_{2}$. Then $\Gamma$ has $K$-fiber $\Gamma_{K}$ because each $X_{i}$ is $S$-separated. We take $X_{3}$ to be the normalization of $\Gamma$. The normalization map $X_{3} \rightarrow \Gamma$ is finite since $\Gamma_{K}$ is smooth, so the natural maps $X_{3} \rightrightarrows X_{i}$ induced by the projection maps $\mathrm{pr}_{i}: X_{1} \times_{S} X_{2} \rightrightarrows X_{i}$ for $i=1,2$ are proper and birational. Thus, by Proposition 2.6, $X_{3}$ is an admissible model of $X_{K}$.

Remark 3.2. It is natural to ask if every smooth proper curve $X_{K}$ over $K$ has an admissible model over $R$. Quite generally, we claim that $X_{K}$ has an admissible model over $R$ if and only if every regular proper model $X$ of $X_{K}$ is cohomologically flat (i.e. admissibe). Indeed, if $X$ is any regular proper model of $X_{K}$ and $X^{\prime}$ is an admissible model of $X_{K}$, then by resolution of singularities and the argument of Lemma 3.1, one knows that there exists a regular model $X^{\prime \prime}$ of $X_{K}$ dominating $X$ and $X^{\prime}$. We conclude from Proposition 2.6 that $X$ must be an admissible model of $X_{K}$.

Thus, if the residue characteristic of $R$ is zero then any regular model of $X_{K}$ over $R$ is admissible, due to Proposition 2.7. On the other hand, if the residue characteristic of $R$ is $p>0$ then by [31, 9.4.3 a)], there exist regular proper curves $X$ that are not cohomologically flat. Nonetheless, "most" curves of interest do have an admissible model, as the Proposition below shows. 
Proposition 3.3. Let $X_{K}$ be a smooth connected and proper curve over $K$ with a $K^{\prime}$-rational point for some tamely ramified extension $K^{\prime}$ of $K$. Then every regular proper model of $X_{K}$ over $R$ is admissible; in particular, $X_{K}$ has an admissible model over $R$.

Proof. Let $K^{\prime}$ be any finite extension of $K$, with ramification index $e$. If $X_{K}$ has a $K^{\prime}$-point, then we claim that any regular proper model of $X_{K}$ over $R$ has an irreducible component in the closed fiber of geometric multiplicity dividing $e$. Proposition 3.3 follows from this, by applying Proposition 2.7 (and using the existence of a regular proper model). To justify the claim, write $v$ for the valuation on $K$ associated to $R$ and choose an extension $v^{\prime}$ of $v$ to $K^{\prime}$. Denote by $R^{\prime}$ the valuation ring of $v^{\prime}$, so $R \rightarrow R^{\prime}$ is a local map of discrete valuation rings with ramification index $e\left(v^{\prime} \mid v\right)=e$ (note that in general, the integral closure of $R$ in $K^{\prime}$ need not be local). Let $X^{\prime}$ be a resolution of singularities of the base change $X \times{ }_{R} R^{\prime}$, and denote by $\rho: X^{\prime} \rightarrow X$ the natural map. As $X^{\prime}$ is proper, the valuative criterion for properness ensures that the $K^{\prime}$-point of $X$ extends to an $R^{\prime}$-point of $X^{\prime}$; in other words, the regular $R^{\prime}$-scheme $X^{\prime}$ has a section. This section must have image in the smooth locus (by [6, $\S 3.1$, Proposition 2]) so we conclude that there is an irreducible component in the closed fiber of $X^{\prime}$ with geometric multiplicity 1 . Letting $\xi^{\prime}$ be the generic point of this component and writing $\xi=\rho\left(\xi^{\prime}\right)$ for the image of $\xi^{\prime}$ in $X$, we then have a canonical local map of discrete valuation rings $\mathscr{O}_{X, \xi} \rightarrow \mathscr{O}_{X^{\prime}, \xi^{\prime}}$. Fixing an algebraic closure $\bar{k}$ of the residue field of $R$ and applying $\otimes_{R} \bar{k}$, we conclude that the length of $\mathscr{O}_{X, \xi} \otimes_{R} \bar{k}$ divides the length of $\mathscr{O}_{X^{\prime}, \xi^{\prime}} \otimes_{R} \bar{k}$. But by our choice of $\xi$, we know that the length of $\mathscr{O}_{X^{\prime}, \xi^{\prime}} \otimes_{R^{\prime}} \bar{k}$ is 1 , and it follows that the length of $\mathscr{O}_{X^{\prime}, \xi^{\prime}} \otimes_{R} \bar{k}$ is just the length of $R^{\prime} \otimes_{R} k$, which is $e$. Thus, the closure of $\xi$ in $X$ is an irreducible component of the closed fiber with geometric multiplicity that divides $e$, giving the claim.

Now suppose that $f_{K}: X_{K} \rightarrow Y_{K}$ is any finite morphism of smooth proper curves over $K$. A standard argument with resolution of singularities and closures of graphs, as in the proof of Lemma 3.1, shows that one can always find regular proper models $X$ and $Y$ of $X_{K}$ and $Y_{K}$ and a morphism $f: X \rightarrow Y$ recovering $f_{K}$ on generic fibers. It is natural to ask if this can be done with $f$ finite as well. As Abhyankar showed [1], the answer is negative in general. The problem has been studied by Liu and Lorenzini $[26, \S 6]$, who show that there are local obstructions in certain cases to extending $f_{K}$ to a finite morphism $f$ between regular models, and give a general class of examples of cyclic étale covers $f_{K}$ that do not extend to finite morphisms of regular models. In the positive direction, Liu [25, Theorem 0.2] has shown that one can always extend $f_{K}$ to a finite morphism between (possibly nonregular) semistable models after passing to a finite separable extension $K^{\prime}$ of $K$. Using the flattening techniques of Gruson-Raynaud [32], when $X_{K}$ and $Y_{K}$ have admissible models we can extend $f_{K}$ to a finite morphism of admissible models over $R$ (i.e. we avoid passing to an extension of the base field). More precisely:

Theorem 3.4. Suppose that $X$ and $Y$ are admissible $S$-curves and $f_{K}: X_{K} \rightarrow Y_{K}$ is a finite morphism of their generic fibers. Then there exist admissible models $X^{\prime}$ of $X_{K}$ and $Y^{\prime}$ of $Y_{K}$ which dominate $X$ and $Y$ respectively and a finite morphism $f^{\prime}: X^{\prime} \rightarrow Y^{\prime}$ recovering $f_{K}$ on generic fibers.

Proof. Let $\Gamma_{K} \subseteq X_{K} \times Y_{K}$ be the graph of $f_{K}$. As $Y$ is $S$-separated, the closure $\Gamma$ of $\Gamma_{K}$ in $X \times Y$ has $K$-fiber $\Gamma_{K}$. Let $\widetilde{\Gamma}$ be the normalization of $\Gamma$. Since the map $\widetilde{\Gamma} \rightarrow \Gamma$ is finite (see the proof of Lemma 3.1) it is proper, and clearly $\widetilde{\Gamma}_{K} \simeq \Gamma_{K} \simeq X_{K}$ via the first projection. Thus, composing the normalization map with the projection maps, we obtain a proper birational $S$-morphism $\widetilde{\Gamma} \rightarrow X$ and an $S$-morphism $f: \widetilde{\Gamma} \rightarrow Y$ that recovers $f_{K}$ on generic fibers. By Proposition 2.6, $\widetilde{\Gamma}$ is admissible. 
If $f$ is quasifinite then it is finite and so we are done. However, this may not be the case. Applying [32, Théorème 5.2.2] with $n=1$ to the morphism $f$, which is generically finite, we find that there is a blowup $Y^{\prime \prime}$ of $Y$ such that the strict transform $\widetilde{\Gamma}^{\prime}$ of $\widetilde{\Gamma}$ with respect to $Y^{\prime \prime} \rightarrow Y$ is quasi-finite over $Y^{\prime \prime}$. We let $X^{\prime}$ be the normalization of $\widetilde{\Gamma}^{\prime}$ and $Y^{\prime}$ be the normalization of $Y^{\prime \prime}$; as we saw in the proof of Lemma 3.1, these normalization maps are finite [18, $I V_{2}, 7.8 .6$ (ii)]. The quasi-finite dominant map $X^{\prime} \rightarrow Y^{\prime \prime}$ necessarily factors through $Y^{\prime}$ (by the universal property of normalization), so we obtain a quasi-finite $S$-morphism $f^{\prime}: X^{\prime} \rightarrow Y^{\prime}$. Now $X^{\prime}$ and $Y^{\prime}$ are evidently proper over $S$ and are admissible by Proposition 2.6; moreover, the proper quasi-finite morphism $f^{\prime}$ must be finite, so we are done (see the diagram below).

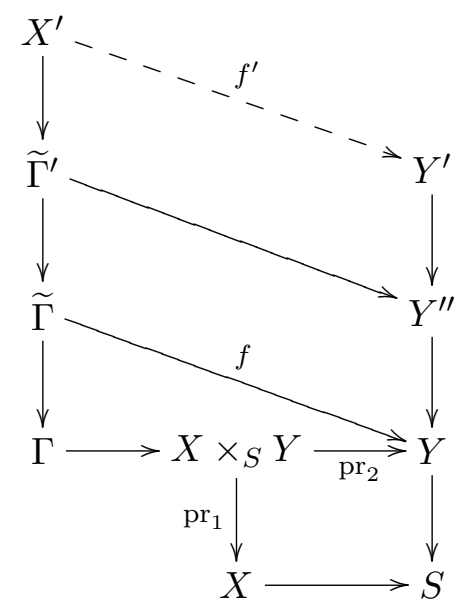

Let $R \rightarrow R^{\prime}$ be a local extension of discrete valuation rings with fraction field extension $K \rightarrow K^{\prime}$ and put $S^{\prime}:=\operatorname{Spec} R^{\prime}$. Suppose that $X_{K}$ has an admissible model $X$ over $S$. By [11, Lemma 2.1.1], if the relative ramification index $e\left(R^{\prime} / R\right)$ is 1 and the residue field extension $k \rightarrow k^{\prime}$ is separable, then $X \times_{S} S^{\prime}$ is an admissible curve, as such base change preserves regularity and normality (since $X_{K}$ is smooth), and cohomology commutes with flat base change. However, if $e\left(R^{\prime} / R\right)>1$ or $k^{\prime} / k$ is not separable then $X \times{ }_{S} S^{\prime}$ may no longer be normal, and its normalization need not be admissible. In general, it is not clear that $X_{K^{\prime}}$ has an admissible model (but see Remark 3.2 and Proposition 3.3), and if it does, how this model is related to $X$. Nevertheless, we can still say something about the behavior of admissible models with respect to base change:

Proposition 3.5. With the notation above, suppose that $X_{K^{\prime}}$ has an admissible model over $S^{\prime}$ and that $K \rightarrow K^{\prime}$ is finite. Then there exists an admissible model $Y$ of $X_{K}$ dominating $X$ such that the normalization of the base change $Y \times_{S} S^{\prime}$ is an admissible model of $X_{K^{\prime}}$ over $S^{\prime}$. Furthermore, $Y$ may be taken to be regular.

Proof. Let $X^{\prime}$ be an admissible model of $X_{K^{\prime}}$ over $S^{\prime}$. As $S^{\prime} \rightarrow S$ is surjective, there is a canonical isomorphism $X^{\prime} \times{ }_{S}$ Spec $K \simeq X_{K^{\prime}}$. By Theorem 3.4, there exist admissible models $Z^{\prime}$ of $X_{K^{\prime}}$ over $S^{\prime}$ and $Z$ of $X_{K}$ over $S$ dominating $X^{\prime}$ and $X$, respectively, and a finite morphism $f: Z^{\prime} \rightarrow Z$ recovering the projection $\left(X^{\prime}\right)_{K} \simeq X_{K^{\prime}} \rightarrow X_{K}$ over $K$. Note that the finiteness of $f$ ensures that the normal scheme $Z^{\prime}$ is the normalization of $Z$ in the function field of $X^{\prime}$. Let $Y \rightarrow Z$ be any proper birational map with $Y$ normal (for example, we could take $Y$ to be a resolution of $Z$ ), and denote by $Y^{\prime}$ the 
normalization of the base change $Y \times{ }_{S} S^{\prime}$. Since $Y^{\prime}$ is normal and has the same function field as $Z^{\prime}$, the map $Y^{\prime} \rightarrow Z$ necessarily factors (uniquely) through $Z^{\prime} \rightarrow Z$. Due to Proposition 2.6, $Y$ is admissible (over $S$ ) and $Y^{\prime}$ is admissible over $S^{\prime}$.

\section{The DUALizing SheAF AND GROThendieCK DUALity}

Recall that a locally finite type map of locally noetherian schemes is said to be Cohen-Macaulay (which we abbreviate to "CM") if it is flat and all fibers are Cohen-Macaulay schemes. The composite of two CM maps is again CM, and the base change of a CM map is CM. Let $X$ and $Z$ be locally noetherian schemes and $f: X \rightarrow Z$ any $\mathrm{CM}$ map with pure relative dimension $n$. Suppose that $Z$ admits a dualizing complex. By [9, Theorem 3.5.1], the relative dualizing complex $f^{!} \mathscr{O}_{Z}$ has a unique nonzero cohomology sheaf, which is in degree $-n$, and we define

$$
\omega_{X / Z}:=H^{-n}\left(f^{!} \mathscr{O}_{Z}\right) .
$$

We call the sheaf $\omega_{X / Z}$ the (relative) dualizing sheaf (for $X$ over $Z$ ). It is flat over $Z$ by $[9$, Theorem 3.5.1], and locally free if and only if the Cohen-Macaulay fibers of $f$ are Gorenstein [19, V, Proposition 9.3, Theorem 9.1] (which holds, for example, when $X$ is regular). Thanks to [9, Theorem 3.6.1], the formation of $\omega_{X / Z}$ is compatible with arbitrary base change on $Z$, and the discussion preceding [9, Corollary 4.4.5] shows moreover that the formation of $\omega_{X / Z}$ is compatible with étale localization on $X$.

Remarks 4.1. We will sometimes write $\omega_{f}$ for $\omega_{X / Z}$, to emphasize its role as relative dualizing sheaf, or to conserve notation as the case may be. In fact, if $Z$ is Gorenstein with finite Krull dimension then $\omega_{X / Z}$ is also a dualizing complex $[19, \mathrm{~V}, \S 2]$ for the abstract scheme $X$. Indeed, this follows from the fact that $f^{!}$carries dualizing complexes for $Z$ to dualizing complexes for $X$, which in turn results from Theorem 8.3 and the Remark following it in $[19, \mathrm{~V}, \S 8]$ and the local nature of being a dualizing complex. We will need this fact later.

We first apply these considerations to the particular situation that $f: X \rightarrow S$ is a relative curve. Since $S=\operatorname{Spec} R$ with $R$ a discrete valuation ring, $S$ is regular and of finite Krull dimension; it follows from $[19, \mathrm{~V}, \S 10]$ that $\mathscr{O}_{S}$ is a dualizing complex for $S$. Moreover, since $X$ is normal and of dimension 2 , it is automatically Cohen-Macaulay by Serre's criterion for normality. In particular, the dualizing complex is a coherent sheaf $\omega_{X / S}$ concentrated in some degree. By our discussion, the sheaf $\omega_{X / S}$ is flat over $S$ and its formation is compatible with arbitrary base change on $S$ and étale localization on $X$.

Remark 4.2. When $X$ is also regular and proper over $S$, there is a more concrete description of $\omega_{X / S}$ that is well-suited to explicit local calculation. Indeed, one knows that $X$ is projective over $S$ [20, Theorem 2.8], so we fix a projective $S$-embedding $i: X \rightarrow \mathbf{P}$ with $\mathbf{P}=\mathbf{P}_{S}^{n}$. Since $f: X \rightarrow S$ is a finite type morphism of regular noetherien schemes, $i$ is a local complete intersection in the sense of [24, Definition 6.3.17]. We may thus consider the canonical sheaf [25, Definition 6.4.7], defined in terms of the exact sequence

$$
0 \longrightarrow \mathscr{C}_{X / \mathbf{P}} \longrightarrow i^{*} \Omega_{\mathbf{P} / S}^{1} \longrightarrow \Omega_{X / S}^{1} \longrightarrow 0
$$

as

$$
\omega_{X / S}^{\mathrm{can}}:=\operatorname{det}\left(\mathscr{C}_{X / \mathbf{P}}\right)^{\vee} \otimes_{\mathscr{O}_{X}} i^{*} \operatorname{det}\left(\Omega_{\mathbf{P} / S}^{1}\right)
$$


By $\left[9,(2.5 .1)\right.$, Corollary 3.5.2] (or [24, Theorem 6.4.32]), there is a canonical isomorphism $\omega_{X / S} \simeq$ $\omega_{X / S}^{\text {can }}$. Although we do not explicitly need this fact, it will be used implicitly several times in what follows (mainly to ensure that what we do regarding the dualizing sheaf is compatible with the work of other authors).

A basic property of the relative dualizing sheaf that will play a crucial role in what we do (and which justifies the name "dualizing") is the following:

Lemma 4.3. Let $X$ be a relative $S$-curve. Then the canonical maps

$$
\omega_{X / S} \longrightarrow \mathbf{R} \mathscr{H} \text { om }{ }_{X}\left(\mathscr{O}_{X}[1], \omega_{X / S}[1]\right)
$$

and

$$
\mathscr{O}_{X}[1] \longrightarrow \mathbf{R} \mathscr{H} \text { om } \bullet_{X}\left(\omega_{X / S}, \omega_{X / S}[1]\right)
$$

are quasi-isomorphisms.

Proof. The first isomorphism is obvious (for any $\mathscr{O}_{X}$-module in the role of $\omega_{X / S}$ ) since $\mathscr{H}$ om $m_{X}\left(\mathscr{O}_{X}, \cdot\right.$ ) is naturally isomorphic to the identity functor. By viewing $\omega_{X / S}$ as $\mathbf{R} \mathscr{H} o m_{X}^{\bullet}\left(\mathscr{O}_{X}[1], \omega_{X / S}[1]\right)$, our assertion for the second map is precisely the claim that the natural double duality map $[9,(1.3 .20)]$

$$
\mathscr{O}_{X}[1] \longrightarrow \mathbf{R} \mathscr{H} \circ m_{X}^{\bullet}\left(\mathbf{R} \mathscr{H} \circ m_{X}^{\bullet}\left(\mathscr{O}_{X}[1], \omega_{X / S}[1]\right), \omega_{X / S}[1]\right)
$$

is a quasi-isomorphism, which is indeed the case as $\omega_{X / S}[1]$ is a dualizing complex for the abstract scheme $X$; see Remarks 4.1 .

Deligne and Rapoport [15, I, §2.1.1] call $\omega_{X / S}$ the sheaf of "regular differentials." This terminology is partially justified since $\omega_{X / S}$ is a subsheaf of the sheaf of differential 1-forms on the generic fiber of $X$. Indeed, let $i: X_{K} \hookrightarrow X$ be the inclusion of the smooth generic fiber into $X$. Since $\omega_{X / S}$ is $S$-flat, the natural map $\omega_{X / S} \rightarrow i_{*} i^{*} \omega_{X / S}$ is injective. Moreover, the general theory of the dualizing sheaf provides a canonical isomorphism $i^{*} \omega_{X / S} \simeq \Omega_{X_{K} / K}^{1}$, so we have an injective map of $\mathscr{O}_{X}$-modules

$$
\omega_{X / S} \hookrightarrow i_{*} \Omega_{X_{K} / K}^{1}
$$

We will see later in Proposition 5.1 that more is true: in fact, the canonical map $\Omega_{X / S}^{1} \rightarrow i_{*} \Omega_{X_{K} / K}^{1}$ uniquely factors through (4.2).

We now wish to apply Grothendieck duality to proper relative curves and to proper (e.g., finite) morphisms of relative curves. Quite generally, if $\rho: X \rightarrow Y$ is any proper map of noetherian schemes and $Y$ admits a dualizing complex, then Grothendieck's theory provides a natural transformation of functors (see $[9, \S 3.4]$ )

$$
\operatorname{Tr}_{\rho}: \mathbf{R} \rho_{*} \rho^{!} \rightarrow 1
$$

on the bounded below derived category $\mathbf{D}_{c}^{+}(Y)$. If $\mathscr{G} \bullet$ is any bounded below complex of abelian sheaves on $Y$ having coherent cohomology, and $\mathscr{F} \bullet$ any bounded above complex on $X$ with quasi-coherent cohomology, then (4.3) yields a natural map

$$
\mathbf{R} \rho_{*} \mathbf{R} \mathscr{H} \circ m_{X}^{\bullet}\left(\mathscr{F} \bullet, \rho^{!} \bullet\right) \longrightarrow \mathbf{R} \mathscr{H} o m_{Y}^{\bullet}\left(\mathbf{R} \rho_{*} \mathscr{F} \bullet, \mathscr{G} \bullet\right)
$$

which by Grothendieck-Serre duality [9, Theorem 3.4.4] is a quasi-isomorphism. 
In particular, if $Y$ is a regular scheme with finite Krull dimension and $\rho$ has pure relative dimension 1, then for $\omega_{X / Y}:=H^{-1}\left(\rho^{!} \mathscr{O}_{Y}\right)$ Grothendieck's trace map (4.3) induces a unique trace map

$$
\gamma_{f}: R^{1} \rho_{*}\left(\omega_{X / Y}\right) \rightarrow \mathscr{O}_{Y}
$$

whose formation commutes with arbitrary base change $Y^{\prime} \rightarrow Y$ with $Y^{\prime}$ another regular scheme with finite Krull dimension [9, Theorem 3.6.5], and we have:

Theorem 4.4. Let $f: X \rightarrow S$ be any proper relative curve. Then the natural maps of free $R$-modules

$$
H^{0}\left(X, \omega_{X / S}\right) \longrightarrow H^{1}\left(X, \mathscr{O}_{X}\right)^{\vee} \text { and } H^{0}\left(X, \mathscr{O}_{X}\right) \longrightarrow H^{1}\left(X, \omega_{X / S}\right)^{\vee}
$$

induced by cup-product and the trace map $\gamma_{f}$ are isomorphisms. Furthermore, there are short exact sequences of $R$-modules

$$
0 \longrightarrow \operatorname{Ext}_{R}^{1}\left(H^{1}\left(X, \mathscr{O}_{X}\right)_{\text {tors }}, R\right) \longrightarrow H^{1}\left(X, \omega_{X / S}\right) \longrightarrow H^{0}\left(X, \mathscr{O}_{X}\right)^{\vee} \longrightarrow 0
$$

and

$$
0 \longrightarrow \operatorname{Ext}_{R}^{1}\left(H^{1}\left(X, \omega_{X / S}\right)_{\text {tors }}, R\right) \longrightarrow H^{1}\left(X, \mathscr{O}_{X}\right) \longrightarrow H^{0}\left(X, \omega_{X / S}\right)^{\vee} \longrightarrow 0 .
$$

In particular the natural maps

$$
H^{1}\left(X, \omega_{X / S}\right) \longrightarrow H^{0}\left(X, \mathscr{O}_{X}\right)^{\vee} \quad \text { and } \quad H^{1}\left(X, \mathscr{O}_{X}\right) \longrightarrow H^{0}\left(X, \omega_{X / S}\right)^{\vee}
$$

induced by cup-product and the trace map are isomorphisms of free $R$-modules if and only if $f$ is cohomologically flat in dimension 0.

Proof. Setting $\mathscr{G}^{\bullet}=\mathscr{O}_{S}$ and $\mathscr{F}^{\bullet}=\mathscr{O}_{X}$ (thought of as complexes in degree zero) in (4.4) and using Lemma 4.3 gives a natural quasi-isomorphism

$$
\mathbf{R} \Gamma\left(X, \omega_{X / S}[1]\right) \simeq \mathbf{R} \operatorname{Hom}_{R}^{\bullet}\left(\mathbf{R} \Gamma\left(X, \mathscr{O}_{X}\right), R\right) .
$$

Applying $H^{-1}$ and using the spectral sequence

$$
E_{2}^{m, n}=\operatorname{Ext}_{R}^{m}\left(H^{-n}\left(X, \mathscr{O}_{X}\right), R\right) \Longrightarrow H^{m+n}\left(\mathbf{R} \operatorname{Hom}_{R}^{\bullet}\left(\mathbf{R} \Gamma\left(X, \mathscr{O}_{X}\right), R\right)\right),
$$

(whose only nonzero terms occur when $m=0,1$ and $n=0,-1$ ) we deduce that the natural map

$$
H^{0}\left(X, \omega_{X / S}\right) \longrightarrow \operatorname{Hom}\left(H^{1}\left(X, \mathscr{O}_{X}\right), R\right)
$$

is an isomorphism. Similarly, by applying $H^{0}$ and recalling that the inclusion of the torsion submodule $H^{1}\left(X, \mathscr{O}_{X}\right)_{\text {tors }} \hookrightarrow H^{1}\left(X, \mathscr{O}_{X}\right)$ induces an isomorphism on Ext ${ }^{1}$ 's, we obtain (4.7). The same argument with $\mathscr{F}^{\bullet}=\omega_{X / S}$ and $\mathscr{G} \bullet=\mathscr{O}_{S}$ yields (again using the identification of Lemma 4.3) the second map in (4.6) and the short exact sequence (4.8).

To know that the resulting maps coincide with the natural ones obtained via cup product and the trace map $\gamma_{f}$, one proceeds as in the proof of [9, Theorem 5.1.2]. Finally, we know that $H^{0}\left(X, \mathscr{O}_{X}\right)$ and $H^{0}\left(X, \omega_{X / S}\right)$ are free since $f$ is flat and $\omega_{X / S}$ is $S$-flat, and since the fibers of $f$ are of pure dimension 1 the $R$-modules $H^{i}\left(X, \mathscr{O}_{X}\right), H^{i}\left(X, \omega_{X / S}\right)$ are both zero for $i>1$ (by the theorem on formal functions). Due to Lemma 2.1, the $R$-module $H^{1}\left(X, \mathscr{O}_{X}\right)$ is free if and only if $f$ is cohomologically flat in dimension zero. 
Now suppose that $\rho: X \rightarrow Y$ is a proper map of relative $S$-curves. We will work out some consequences of Grothendieck duality for $\rho$. Denoting by $f: X \rightarrow S$ and $g: Y \rightarrow S$ the structural morphisms, by definition of the relative dualizing sheaf (4.1) we have quasi-isomorphisms

$$
\omega_{X / S}[1] \simeq f^{!} \mathscr{O}_{S} \text { and } \omega_{Y / S}[1] \simeq g^{!} \mathscr{O}_{S} .
$$

Since $\rho$ is an $S$-morphism, we have $f=g \circ \rho$, and there is a natural isomorphism of functors $f^{!} \simeq \rho^{!} g^{!}$ given as in $[9,(3.3 .14)]$. Thus, we have a quasi-isomorphism

$$
\omega_{X / S}[1] \simeq f^{!} \mathscr{O}_{S} \simeq \rho^{!} g^{!} \mathscr{O}_{S} \simeq \rho^{!} \omega_{Y / S}[1] .
$$

and Grothendieck-Serre duality reads

$$
\mathbf{R} \rho_{*} \mathbf{R} \mathscr{H} o m_{X}^{\bullet}\left(\mathscr{F}^{\bullet}, \omega_{X / S}[1]\right) \simeq \mathbf{R} \mathscr{H} o m_{Y}^{\bullet}\left(\mathbf{R} \rho_{*} \mathscr{F} \bullet, \omega_{Y / S}[1]\right)
$$

for any bounded above complex of sheaves $\mathscr{F}^{\bullet}$ on $X$ with quasi-coherent cohomology. By the definition of the duality isomorphism (4.4), the map (4.10) is induced by the natural trace map in the derived category

$$
\mathbf{R} \rho_{*} \omega_{X / S} \longrightarrow \omega_{Y / S}
$$

obtained from (4.9) and Grothendieck's trace map (4.3). If we apply $H^{-1}$ to the composite of (4.11) and the natural map $\rho_{*} \omega_{X / S} \rightarrow \mathbf{R} \rho_{*} \omega_{X / S}$, we get a canonical $\mathscr{O}_{Y}$-linear trace map on relative dualizing sheaves

$$
\rho_{*} \omega_{X / S} \longrightarrow \omega_{Y / S} .
$$

We know from Lemma 4.3 that via the functor $\mathbf{R} \mathscr{H}$ om $m_{Y}^{\bullet}\left(\cdot, \omega_{Y}[1]\right)$, the complexes $\mathscr{O}_{Y}[1]$ and $\omega_{Y / S}$ are dual. Due to (4.10), the same is true of $\mathbf{R} \rho_{*} \mathscr{O}_{X}$ and $\mathbf{R} \rho_{*} \omega_{X / S}$. This duality furthermore interchanges pullback and trace:

Proposition 4.5. Let $\rho: X \rightarrow Y$ be a proper map of relative $S$-curves. Then the trace morphism on relative dualizing sheaves (4.12) is dual (via the identifications of Lemma 4.3) to the canonical pullback map $\mathscr{O}_{Y} \longrightarrow \rho_{*} \mathscr{O}_{X}$ on functions. If $\rho$ is in addition finite and generically étale, then there exist unique $\mathscr{O}_{Y}$-linear trace and pullback maps

$$
\begin{gathered}
\rho_{*}: \rho_{*} \mathscr{O}_{X} \longrightarrow \mathscr{O}_{Y} \\
\rho^{*}: \omega_{Y / S} \longrightarrow \rho_{*} \omega_{X / S}
\end{gathered}
$$

which are dual and which coincide with the evident trace and pullback maps on the smooth $K$-fibers. In particular, when $X$ and $Y$ are smooth and $\rho$ is finite étale, these maps recover the usual pullback and trace maps on functions and differential forms (cf. Proposition 5.1).

Proof. Applying $\mathbf{R} \mathscr{H}$ om ${ }_{Y}\left(\cdot, \omega_{Y}\right)$ to the pullback map $\mathscr{O}_{Y} \rightarrow \rho_{*} \mathscr{O}_{X}$ and using (4.10) gives a map in the derived category

$$
\mathbf{R} \rho_{*} \mathbf{R} \mathscr{H} o m_{X}^{\bullet}\left(\mathscr{O}_{X}, \omega_{X / S}\right) \simeq \mathbf{R} \mathscr{H} o m_{Y}^{\bullet}\left(\mathbf{R} \rho_{*} \mathscr{O}_{X}, \omega_{Y / S}\right) \longrightarrow \mathbf{R} \mathscr{H} o m_{Y}^{\bullet}\left(\mathscr{O}_{Y}, \omega_{Y}\right)
$$

so by Lemma 4.3 we obtain a map $\mathbf{R} \rho_{*} \omega_{X / S} \rightarrow \omega_{Y / S}$ which by definition of the map (4.4) is none other than the trace map (4.11). If $\rho$ is generically étale, then on $K$-fibers we claim that the trace map (4.12) is identified with the usual trace map on differential forms. To check this, by working over 
a Zariski dense open in $Y_{K}$ over which $\rho_{K}$ is finite étale, we may reduce to the case that $\rho_{K}$ is finite étale, and we wish to show in this context that (4.12) coincides with the map

$$
\rho_{K *} \Omega_{X_{K} / K}^{1} \simeq \rho_{K *} \mathscr{O}_{X_{K}} \otimes \Omega_{Y_{K} / K}^{1} \longrightarrow \Omega_{Y_{K} / K}^{1}
$$

that sends a section $b \otimes \eta$ to $\operatorname{Tr}(b) \cdot \eta$, where $\operatorname{Tr}: \rho_{K *} \mathscr{O}_{X_{K}} \rightarrow \mathscr{O}_{Y_{K}}$ is the usual trace morphism associated to the finite locally free extension of algebras $\mathscr{O}_{Y_{K}} \rightarrow \rho_{K *} \mathscr{O}_{X_{K}}$. This follows easily from the construction of Grothendieck's trace map in the finite étale case; see for instance (2.7.10) and the description of (2.7.37) in [9], or [19, III, §6].

Suppose now that $\rho: X \rightarrow Y$ is finite and generically étale. We first claim that we have a natural $\mathscr{O}_{Y}$-linear trace map (4.13). If $\rho$ is in addition flat, then this is clear, as we take $\rho_{*}$ to be the usual trace map on functions $\operatorname{Tr}$ as above. In general, since $Y$ is normal, $\rho$ must be finite flat over the generic points of the special fiber of $Y$, so we can find a Zariski open subset $V \subseteq Y$ with scheme-theoretic preimage $U:=\rho^{-1} V$ such that $\rho_{U}: U \rightarrow V$ is finite flat and the complements of $V$ in $Y$ and of $U$ in $X$ consist of finitely many points of codimension 2 (necessarily in the closed fibers). Since $X$ and $Y$ are normal, we may thus define (4.13) to be the composite

$$
\rho_{*} \mathscr{O}_{X} \stackrel{\simeq}{\longrightarrow} \rho_{*} i_{*} \mathscr{O}_{U}=j_{*} \rho_{U *} \mathscr{O}_{U} \stackrel{j_{*}\left(\rho_{U *}\right)}{\longrightarrow} j_{*} \mathscr{O}_{V} \stackrel{\simeq}{\longleftarrow} \mathscr{O}_{Y}
$$

Observe that on generic fibers, the trace map (4.15) recovers the usual trace morphism associated to the finite flat and generically étale morphism $f_{K}$. In particular, the preceding construction is independent of the choice of $V$.

To obtain the desired pullback map (4.14) on dualizing sheaves, we apply $\mathbf{R} \mathscr{H} o m_{Y}^{\bullet}\left(\cdot, \omega_{Y / S}\right)$ to the morphism (4.13), which yields a derived category map

$$
\mathbf{R} \mathscr{H} o m_{Y}^{\bullet}\left(\mathscr{O}_{Y}, \omega_{Y / S}\right) \longrightarrow \mathbf{R} \mathscr{H} o m_{Y}^{\bullet}\left(\mathbf{R} \rho_{*} \mathscr{O}_{X}, \omega_{Y / S}\right) \simeq \mathbf{R} \rho_{*} \mathbf{R} \mathscr{H} o m_{X}^{\bullet}\left(\mathscr{O}_{X}, \omega_{X / S}\right)
$$

By Lemma 4.3 we get a derived category map $\omega_{Y / S} \rightarrow \mathbf{R} \rho_{*} \omega_{X / S}$, and since $\rho$ is finite (so $\rho_{*} \rightarrow \mathbf{R} \rho_{*}$ is a quasi-isomorphism on coherent sheaves) we obtain the claimed pullback map (4.14) on dualizing sheaves by applying $H^{0}$. By construction, the resulting map is dual to the trace map (4.13) on functions.

Let us see that on $K$-fibers (4.14) really is the usual pullback map on differential forms. By shrinking $Y$ if need be, we may assume that $\rho_{K}$ is finite étale. By definition, (4.14) in this case is the map

$$
\Omega_{Y_{K} / K}^{1} \longrightarrow \mathscr{H} \operatorname{om}_{Y_{K}}\left(\mathscr{O}_{Y_{K}}, \Omega_{Y_{K} / K}^{1}\right) \longrightarrow \mathscr{H} \operatorname{om}_{Y_{K}}\left(\rho_{K *} \mathscr{O}_{X_{K}}, \Omega_{Y_{K} / K}^{1}\right) \simeq \rho_{K *} \Omega_{X_{K} / K}^{1}
$$

that sends a section $\eta$ of $\Omega_{Y_{K} / K}^{1}$ to the section $b^{\prime} \mapsto \operatorname{Tr}\left(b^{\prime}\right) \cdot \eta$ of $\operatorname{Hom}_{Y_{K}}\left(\rho_{K_{*}} \mathscr{O}_{X_{K}}, \Omega_{Y_{K} / K}^{1}\right)$. The Grothendieck duality isomorphism

$$
\rho_{K *} \Omega_{X_{K} / K}^{1} \simeq \rho_{K *} \mathscr{O}_{X_{K}} \otimes \Omega_{Y_{K} / K}^{1} \longrightarrow \mathscr{H} 0 m_{Y_{K}}\left(\rho_{K_{*}} \mathscr{O}_{X_{K}}, \Omega_{Y_{K} / K}^{1}\right)
$$

in this context is precisely the map sending a section $b \otimes \eta$ of $\rho_{K *} \mathscr{O}_{X_{K}} \otimes \Omega_{Y_{K} / K}^{1}$ to the section $\left(b^{\prime} \mapsto \operatorname{Tr}\left(b b^{\prime}\right) \cdot \eta\right)$ of $\mathscr{H} o m_{Y_{K}}\left(\rho_{K *} \mathscr{O}_{X_{K}}, \Omega_{Y_{K} / K}^{1}\right)$; see (2.7.10) and (2.7.9) in [9], and compare to [19, III, pg. 187]. Thus, the resulting pullback map on dualizing sheaves (4.14) is the map sending the section $\eta$ of $\Omega_{Y_{K} / K}^{1}$ to the section $1 \otimes \eta$ of $\rho_{K *} \mathscr{O}_{X_{K}} \otimes \Omega_{Y_{K} / K}^{1} \simeq \rho_{K *} \Omega_{X_{K} / K}^{1}$, and is hence the usual pullback map on differentials as claimed. 
We end this section by recording a useful consequence of rational singularities:

Proposition 4.6. Let $X$ be a proper relative $S$-curve, and let $\rho: X^{\prime} \rightarrow X$ be any proper birational morphism with $X^{\prime}$ normal. If $X$ has rational singularities, then the trace map $\rho_{*}: \rho_{*} \omega_{X^{\prime} / S} \rightarrow \omega_{X / S}$ of (4.12) is an isomorphism.

Proof. By the characterization of rational singularities in Remark 2.3, the natural map $\mathscr{O}_{X} \rightarrow \mathbf{R} \rho_{*} \mathscr{O}_{X^{\prime}}$ is a quasi-isomorphism, so substituting $\mathscr{G}^{\bullet}=\omega_{X / S}$ and $\mathscr{F}^{\bullet}=\mathscr{O}_{X^{\prime}}$ in (4.4) and using Lemma 4.3, we obtain a quasi-isomorphism

$$
\mathbf{R} \rho_{*} \omega_{X^{\prime} / S} \stackrel{\simeq}{\longrightarrow} \omega_{X / S}
$$

By definition of the duality isomorphism (4.4), the map (4.17) is none other than the trace map (4.11). Applying $H^{0}$ to (4.17) thus completes the proof.

\section{Cohomology of Regular differentials}

Fix a relative curve $f: X \rightarrow S$. In this section we study the two-term complex of coherent $\mathscr{O}_{X^{-}}$ modules whose hypercohomology will be a suitable replacement for the relative de Rham cohomology of $X$ over $S$ and, when $X$ is admissible, will provide the sought-after canonical integral structure on $H_{\mathrm{dR}}^{1}\left(X_{K} / K\right)$. In order to define this complex, we need the following well-known fact:

Proposition 5.1. Let $i: X_{K} \hookrightarrow X$ be the inclusion. Then the canonical pullback map of differentials $\Omega_{X / S}^{1} \rightarrow i_{*} \Omega_{X_{K} / K}^{1}$ factors through the inclusion $\omega_{X / S} \hookrightarrow i_{*} \Omega_{X_{K} / K}^{1}$ of (4.2) via a unique $\mathscr{O}_{X}$-linear homomorphism

$$
c_{X / S}: \Omega_{X / S}^{1} \longrightarrow \omega_{X / S} .
$$

Moreover, the restriction of $c_{X / S}$ to any $S$-smooth open subset of $X$ is an isomorphism.

Proof. Uniqueness is clear, as $\omega_{X / S}$ is a subsheaf of $i_{*} \Omega_{X_{K} / K}^{1}$. When $X$ is regular, existence follows immediately from Remark 4.2 and [24, Corollary 6.4.13]. In general, let $\rho: X^{\prime} \rightarrow X$ be a resolution of singularities. We then define $c_{X / S}$ to be the composite

$$
\Omega_{X / S}^{1} \longrightarrow \rho_{*} \Omega_{X^{\prime} / S}^{1} \stackrel{\rho_{*} c_{X^{\prime} / S}}{\longrightarrow} \rho_{*} \omega_{X^{\prime} / S} \stackrel{(4.12)}{\longrightarrow} \omega_{X / S},
$$

where the first map is the usual pullback map on differential 1-forms attached to $\rho$. Since $\rho$ is an isomorphism over the $S$-smooth locus of $X$, we deduce that the same is true for $c_{X / S}$ from the corresponding statement for $c_{X^{\prime} / S}$.

Remark 5.2. In general, the morphism $c_{X / S}: \Omega_{X / S}^{1} \rightarrow \omega_{X / S}$ is neither injective nor surjective, and the kernel and cokernel of $c_{X / S}$ encode certain arithmetic information about $X$. Specifically, when the residue field of $R$ is perfect and $X$ is regular, Bloch shows [5, Theorem 2.3] that the Euler characteristic of the two-term (generically exact) complex defined by $c_{X / S}$ is equal to $-\operatorname{Art}(X / S)$, where $\operatorname{Art}(X / S)$ is the Artin conductor of $X$ over $S$; see $\S 6$.

Thanks to Proposition 5.1, we have a two-term $\mathscr{O}_{S}$-linear complex (of $\mathscr{O}_{S}$-flat $\mathscr{O}_{X}$-modules) concentrated in degrees 0 and 1

$$
\omega_{X / S}^{\bullet}:=\mathscr{O}_{X} \stackrel{d_{S}}{\longrightarrow} \omega_{X / S},
$$


with $d_{S}$ the composite of the map $c_{X / S}$ and the universal derivation $\mathscr{O}_{X} \rightarrow \Omega_{X / S}^{1}$. Since $c_{X / S}$ is an isomorphism over the $S$-smooth locus of $f$ in $X$, we see that on the generic fiber of $X$ the complex $\omega_{X / S}^{\bullet}$ is the usual de Rham complex of $X_{K}$.

Remark 5.3. Our choice to have $\omega_{X / S}^{\bullet}$ in degrees 0 and 1 reflects our desire to think of it as a replacement for the de Rham complex of $X$ over $S$. This choice will cause a small nuisance when we apply duality theory to $\omega_{X / S}^{\bullet}$, as $f^{!} \mathscr{O}_{S}=\omega_{X / S}[1]$, so we will frequently have to "shift degrees" in arguments. We will sometimes do this implicitly, and in any case without further mention; throughout we follow the conventions of $[9, \S 1.3]$.

For use in what follows, let us record some properties of the complex $\omega_{X / S}^{\bullet}$.

Lemma 5.4. Let $X$ be a relative $S$-curve. Then the natural map

$$
\omega_{X / S}^{\bullet} \longrightarrow \mathbf{R} \mathscr{H} \circ m_{X}^{\bullet}\left(\omega_{X / S}^{\bullet}, \omega_{X / S}[-1]\right)
$$

arising from the two maps in Lemma 4.3 is a quasi-isomorphism. More generally, if $\rho: X \rightarrow Y$ is any finite morphism of relative curves over $S$, then there is a canonical quasi-isomorphism

$$
\mathbf{R} \rho_{*} \omega_{X / S}^{\bullet} \simeq \mathbf{R} \mathscr{H} o m_{Y}^{\bullet}\left(\mathbf{R} \rho_{*} \omega_{X / S}^{\bullet}, \omega_{Y / S}[-1]\right) .
$$

Proof. Denote by $f$ and $g$ the structural morphisms $X \rightarrow S$ and $Y \rightarrow S$, respectively, and recall our notational convention from Remark 4.1. Applying $\mathbf{R} \rho_{*}$ to (5.2) and using Grothendieck duality (4.10), we easily see that the second claim follows from the first.

As for the first claim, the evident exact triangle

$$
\omega_{f}[-1] \longrightarrow \omega_{f}^{\bullet} \longrightarrow \mathscr{O}_{X}
$$

and the map (5.2) fit into a morphism of exact triangles

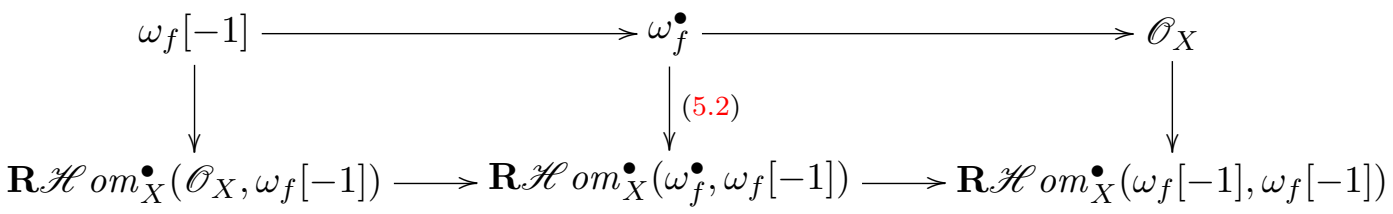

where the bottom row is obtained from the top by applying $\mathbf{R} \mathscr{H} o m_{X}^{\bullet}\left(\cdot, \omega_{f}[-1]\right)$, and the flanking vertical maps are the ones from Lemma 4.3. These flanking maps are quasi-isomorphisms, by Lemma 4.3, so we conclude that the same is true of (5.2), as desired.

Now we show that the complex $\omega_{X / S}^{\bullet}$ is both covariantly and contravariantly functorial in finite generically étale morphisms of relative curves:

Proposition 5.5. Let $\rho: X \rightarrow Y$ be a finite and generically étale morphism of relative S-curves. Then there exist pullback and trace maps of complexes

$$
\omega_{Y / S}^{\bullet} \stackrel{\rho^{*}}{\longrightarrow} \mathbf{R} \rho_{*} \omega_{X / S}^{\bullet} \quad \mathbf{R} \rho_{*} \omega_{X / S}^{\bullet} \stackrel{\rho_{*}}{\longrightarrow} \omega_{Y / S}^{\bullet}
$$

that recover the usual pullback and trace maps on de Rham complexes over the $K$-fibers. Moreover, these maps are dual with respect to the duality functor $\mathbf{R} \mathscr{H}$ om $\bullet_{Y}\left(\cdot, \omega_{Y / S}[1]\right)$. 
Proof. Since $\rho$ is finite, the canonical map $\rho_{*} \omega_{X / S}^{\bullet} \rightarrow \mathbf{R} \rho_{*} \omega_{X / S}^{\bullet}$ is a quasi-isomorphism. Thus, it suffices to construct the desired maps with $\mathbf{R} \rho_{*}$ replaced by $\rho_{*}$. For pullback, we take the usual pullback on functions in degree 0 and the morphism (4.14) in degree 1. Similarly, for trace we take the trace map on dualizing sheaves (4.12) in degree 1 and the map (4.13) in degree 0. That these really yield maps of complexes may be checked at the generic point of the generic fiber of $Y$ (since the sheaves involved are all flat and $\rho_{*}$ is left exact, so preserves injections), where - thanks to our assumption that $\rho$ is generically étale - it follows from Proposition 4.5 and the description on $K$-fibers. The duality statement follows easily from Lemma 5.4 and Proposition 4.5.

We next turn to the study of the hypercohomology of the complex $\omega_{X / S}^{\bullet}$, which when $X$ is admissible will provide the desired canonical integral structure on $H_{\mathrm{dR}}^{1}\left(X_{K} / K\right)$.

Definition 5.6. Let $f: X \rightarrow S$ be a proper relative curve over $S=\operatorname{Spec} R$. We define

$$
H^{i}(X / R):=\mathbf{H}^{i}\left(X, \omega_{X / S}^{\bullet}\right)
$$

Note that $H^{i}(X / R)$ is nonzero only for $0 \leq i \leq 2$. Due to Lemma 5.5, for each $i$ the association $X \rightsquigarrow H^{i}(X / R)$ is both a contravariant functor (via pullback) and a covariant functor (via trace) from the category of proper relative $S$-curves with finite generically étale morphisms to the category of finite $R$-modules. Moreover, as the restriction of $\omega_{X / S}^{\bullet}$ to the generic fiber $X_{K}$ is the usual de Rham complex of $X_{K}$, we have natural isomorphisms

$$
H^{i}(X / R) \otimes_{R} K \simeq H_{\mathrm{dR}}^{i}\left(X_{K} / K\right) .
$$

When $X$ is cohomologically flat (for example, admissible), the $R$-modules $H^{i}(X / R)$ are all free and admit a simple description reminiscent of the structure of the de Rham cohomology of the smooth and proper curve $X_{K}$ :

Proposition 5.7. Suppose that $f: X \rightarrow S$ is a proper and cohomologically flat relative curve. Then there are canonical isomorphisms of free $R$-modules of rank one

$$
H^{0}(X / R) \simeq H^{0}\left(X, \mathscr{O}_{X}\right) \text { and } H^{2}(X / R) \simeq H^{1}\left(X, \omega_{X / S}\right)
$$

and a short exact sequence of finite free $R$-modules

$$
0 \longrightarrow H^{0}\left(X, \omega_{X / S}\right) \longrightarrow H^{1}(X / R) \longrightarrow H^{1}\left(X, \mathscr{O}_{X}\right) \longrightarrow 0
$$

which is functorial (both covariant and contravariant) in finite generically étale morphisms of proper and cohomologically flat curves, and recovers the Hodge filtration of $H_{\mathrm{dR}}^{1}\left(X_{K} / K\right)$ after tensoring with $K$.

Proof. Associated to "la filtration bête" [12] of $\omega_{X / S}^{\bullet}$ is the usual hypercohomology spectral sequence

$$
E_{1}^{i j}=H^{j}\left(X, \omega_{X / S}^{i}\right) \Longrightarrow H^{i+j}(X / R)
$$

which we claim degenerates at the $E_{1}$-stage. To see this, it suffices to show that the differentials

$$
H^{j}\left(X, \mathscr{O}_{X}\right) \stackrel{d_{S}}{\longrightarrow} H^{j}\left(X, \omega_{X / S}\right)
$$

are zero for $j=0,1$ since $E_{1}^{i j}=0$ except possibly for $0 \leq i, j \leq 1$. Since the Hodge to de Rham spectral sequence for $X_{K}$ degenerates at the $E_{1}$-stage [13], the image of $d_{S}$ must be torsion. However, 
$H^{j}\left(X, \omega_{X / S}\right)$ is torsion free for $j=0$ due to $S$-flatness of $\omega_{X / S}$, and for $j=1$ by Theorem 2.1 (since $X$ is cohomologically flat). It follows that the edge maps

$$
H^{0}(X / R) \longrightarrow H^{0}\left(X, \mathscr{O}_{X}\right) \quad \text { and } \quad H^{1}\left(X, \omega_{X / S}\right) \longrightarrow H^{2}(X / R)
$$

are isomorphisms, and that we have an exact sequence (5.6). The flanking $R$-modules in (5.6) are free due to $S$-flatness of $\omega_{X / S}$ and cohomological flatness of $X$ (Lemma 2.1). Thus, $H^{1}(X / R)$ is a free $R$-module as well. By construction and Proposition 5.5, the exact sequence (5.6) is functorial (both covariantly and contravariantly) in finite generically étale $S$-morphisms of proper and cohomologically flat relative curves, and recovers the Hodge filtration of $H_{\mathrm{dR}}^{1}\left(X_{K} / K\right)$ after tensoring with $K$.

To ease notation, we will abbreviate the short exact sequence (5.6) by $H(X / R)$. Thus, $H(X / R)$ gives an integral structure on the 3 -term short exact sequence $H\left(X_{K} / K\right)$ arising from Hodge filtration of $H_{\mathrm{dR}}^{1}\left(X_{K} / K\right)$ as in (1.1).

Applying the functor $\operatorname{Hom}_{R}(\cdot, R)$ to $H(X / R)$, we obtain a sequence $H(X / R)^{\vee}$ of free $R$-modules

$$
0 \longrightarrow H^{1}\left(X, \mathscr{O}_{X}\right)^{\vee} \longrightarrow H^{1}(X / R)^{\vee} \longrightarrow H^{0}\left(X, \omega_{X / S}\right)^{\vee} \longrightarrow 0
$$

which is also short exact.

Proposition 5.8. Let $f: X \rightarrow S$ be a proper and cohomologically flat relative curve. There is a canonical isomorphism of short exact sequences of free $R$-modules

$$
H(X / R) \longrightarrow H(X / R)^{\vee} .
$$

Proof. Applying $\mathbf{R} f_{*}$ to the quasi-isomorphism of exact triangles (5.4) and using Grothendieck duality for $f$, we get a quasi-isomorphism

$$
\mathbf{R} f_{*} \omega_{f}^{\bullet} \stackrel{\simeq}{\mathbf{R}} f_{*} \mathbf{R} \mathscr{H} o m_{X}\left(\omega_{f}^{\bullet}, \omega_{f}[-1]\right) \stackrel{\simeq}{\longrightarrow} \mathbf{R} \mathscr{H} o m_{S}^{\bullet}\left(\mathbf{R} f_{*} \omega_{f}^{\bullet}, \mathscr{O}_{S}\right)[-2]
$$

that is compatible with the evident filtrations on both sides arising from (5.4). The spectral sequence

$$
E_{2}^{m, n}=\operatorname{Ext}_{R}^{m}\left(\mathbf{H}^{-n}\left(X, \omega_{f}^{\bullet}\right), R\right) \Longrightarrow H^{m+n}\left(\mathbf{R} \operatorname{Hom}_{R}^{\bullet}\left(\mathbf{R} \Gamma\left(X, \omega_{f}^{\bullet}\right), R\right)\right)
$$

is compatible with the filtrations in (5.4), so since

$$
\operatorname{Ext}_{R}^{1}\left(\mathbf{H}^{2}\left(X, \omega_{f}^{\bullet}\right), R\right)=0
$$

by Proposition 5.7, we get the claimed isomorphism $H^{1}(X / R) \rightarrow H^{1}(X / R)^{\vee}$, compatibly with the filtrations.

Remark 5.9. There is a more "concrete" description of the isomorphism (5.7). Indeed, on the one hand, for any two bounded below complexes $\mathscr{F} \bullet$ and $\mathscr{G} \bullet$ of $\mathscr{O}_{X}$-modules, abstract nonsense with canonical flasque resolutions provides a bi-functorial map

$$
\mathbf{H}^{m}\left(X, \mathscr{F}^{\bullet}\right) \otimes_{\Gamma\left(X, \mathscr{O}_{X}\right)} \mathbf{H}^{n}\left(X, \mathscr{G}^{\bullet}\right) \longrightarrow \mathbf{H}^{m+n}\left(\operatorname{Tot}^{\oplus}\left(\mathscr{F} \bullet \otimes_{\mathscr{O}_{X}} \mathscr{G} \bullet\right)\right) .
$$

On the other hand, there is a natural map of complexes

$$
\operatorname{Tot}^{\oplus}\left(\omega_{X / S}^{\bullet} \otimes_{\mathcal{O}_{X}} \omega_{X / S}^{\bullet}\right) \longrightarrow \omega_{X / S}^{\bullet}
$$


defined by the canonical maps

$$
\omega_{X / S}^{i} \otimes_{\mathscr{O}_{X}} \omega_{X / S}^{j} \longrightarrow \omega_{X / S}^{i+j}
$$

which are zero for $i+j>1$ and are given by multiplication for $i+j \leq 1$. Applying (5.9) with $\mathscr{F}^{\bullet}=\mathscr{G}^{\bullet}=\omega_{X / S}^{\bullet}$ and composing with the map on hypercohomology induced by (5.10) yields a natural $R$-linear "cup product" map

$$
H^{1}(X / R) \otimes_{R} H^{1}(X / R) \longrightarrow H^{2}(X / R) .
$$

That this map recovers the usual cup product after extending scalars to $K$ follows easily from the definition of the maps (5.11) and the construction of cup products in de Rham cohomology. Composing (5.12) with the edge map isomorphism $H^{2}(X / R) \simeq H^{1}\left(X, \omega_{X / S}\right)$ followed by Grothendieck's trace map (4.5) gives an $R$-bilinear pairing on $H^{1}(X / R)$ which is easily seen to induce a map of short exact sequences $H(X / R) \rightarrow H(X / R)^{\vee}$. That this is the same map as (5.7) may be checked after extending scalars to $K$, where it follows from the classical description of Grothendick duality in terms of cup-product and trace (see, for example, the proof of Theorem 5.1.2 in [9]).

Before getting to the main result of this section, let us address the following natural question. Suppose that $R \rightarrow R^{\prime}$ is a finite local extension of discrete valuation rings with associated fraction field extension $K \rightarrow K^{\prime}$ that is separable. Let $X$ be a proper and cohomologically flat relative curve over $S^{\prime}:=\operatorname{Spec} R^{\prime}$. Considering $X$ as a proper relative curve over $S$, the characterization of cohomological flatness in Lemma 2.1 shows that $X$ is also cohomologically flat over $S$. Note that there is a canonical identification of generic fibers $X \otimes_{R^{\prime}} K^{\prime} \simeq X \otimes_{R} K$, so we may speak of the generic fiber $X_{K}$ of $X$. Since $K^{\prime} / K$ is separable (i.e. smooth), the canonical surjective map $\Omega_{X_{K} / K}^{1} \rightarrow \Omega_{X_{K} / K^{\prime}}^{1}$ is an isomorphism and induces a natural isomorphism

$$
H_{\mathrm{dR}}^{1}\left(X_{K} / K\right) \simeq H_{\mathrm{dR}}^{1}\left(X_{K} / K^{\prime}\right),
$$

compatibly with Hodge filtrations. It thus makes sense to ask how the lattices $H^{1}(X / R)$ and $H^{1}\left(X / R^{\prime}\right)$ are related inside $H_{\mathrm{dR}}^{1}\left(X_{K} / K\right)$.

Theorem 5.10. With the notation above, let $\Delta \subseteq R^{\prime}$ be the different of the extension $R \rightarrow R^{\prime}$. As short exact sequences of lattices inside the Hodge filtration $H\left(X_{K} / K\right)$, there are canonical $R^{\prime}$ linear inclusions $\Delta H(X / R) \hookrightarrow H\left(X / R^{\prime}\right)$ and $H\left(X / R^{\prime}\right) \hookrightarrow H(X / R)$. Via these inclusions of lattices, $H^{0}\left(X, \omega_{X / S^{\prime}}\right)$ is identified with $\Delta H^{0}\left(X, \omega_{X / S}\right)$, and the induced natural maps of $R^{\prime}$-modules

$$
\frac{H^{1}\left(X / R^{\prime}\right)}{\Delta H^{1}(X / R)} \stackrel{\simeq}{\longrightarrow} \frac{H^{1}\left(X, \mathscr{O}_{X}\right)}{\Delta H^{1}\left(X, \mathscr{O}_{X}\right)} \quad \text { and } \quad \frac{H^{0}\left(X, \omega_{X / S}\right)}{\Delta H^{0}\left(X, \omega_{X / S}\right)} \stackrel{\simeq}{\longrightarrow} \frac{H^{1}(X / R)}{H^{1}\left(X / R^{\prime}\right)}
$$

are isomorphisms.

Proof. Let us denote by $f^{\prime}: X \rightarrow S^{\prime}$ the structural morphism, and by $g: S^{\prime} \rightarrow S$ the natural map. By [9, Theorem 4.3.3], there is a canonical isomorphism of $\mathscr{O}_{X}$-modules

$$
\omega_{X / S} \simeq \omega_{X / S^{\prime}} \otimes_{\mathscr{O}_{X}} f^{\prime *}\left(g^{!} \mathscr{O}_{S}\right)
$$

Since $R \rightarrow R^{\prime}$ is finite, we have (canonically)

$$
g^{!} \mathscr{O}_{S} \simeq \mathscr{H} \operatorname{om}_{S}\left(g_{*} \mathscr{O}_{S^{\prime}}, \mathscr{O}_{S}\right)
$$


Now by definition of the different, the fractional $R^{\prime}$-ideal $\Delta^{-1} \subseteq K^{\prime}$ is

$$
\Delta^{-1}:=\left\{\alpha \in K^{\prime}: \operatorname{Tr}_{K^{\prime} / K}\left(\alpha R^{\prime}\right) \subseteq R\right\}
$$

which is mapped isomorphically (as $R^{\prime}$-module) onto $\operatorname{Hom}_{R}\left(R^{\prime}, R\right)$ via the map $\alpha \mapsto \operatorname{Tr}_{K^{\prime} / K}(\alpha(\cdot))$. Thus, denoting by $\widetilde{\Delta}$ the ideal sheaf of $\mathscr{O}_{S^{\prime}}$ associated to the different $\Delta \subseteq R^{\prime}$, we have a natural isomorphism of $\mathscr{O}_{X}$-modules

$$
\omega_{X / S^{\prime}} \simeq\left(f^{\prime}\right)^{*}(\widetilde{\Delta}) \otimes_{\mathscr{O}_{X}} \omega_{X / S}=\widetilde{\Delta} \otimes_{\mathscr{O}_{S^{\prime}}} \omega_{X / S} \simeq \widetilde{\Delta} \cdot \omega_{X / S}
$$

where the final isomorphism results from the fact that $\omega_{X / S}$ is $S$-flat (hence $S^{\prime}$-flat). The explicit construction of the isomorphism (5.14) given in [24, Theorem 6.4.9] shows that on $K$-fibers, the map (5.15) is the inverse of the canonical isomorphism $\Omega_{X_{K} / K}^{1} \stackrel{\simeq}{\longrightarrow} \Omega_{X_{K} / K^{\prime}}^{1}$. Choosing a generator $\delta$ of $\Delta$, since $\omega_{X / S}$ is $S^{\prime}$-flat we therefore have a commutative diagram of $\mathscr{O}_{X}$-modules

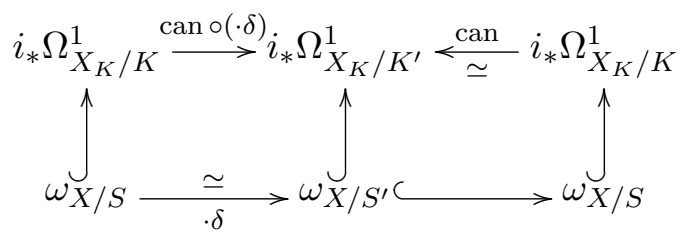

where $i: X_{K} \hookrightarrow X$ is the inclusion of the generic fiber and can is the canonical isomorphism. We claim that this diagram extends to a commutative diagram of two-term complexes:

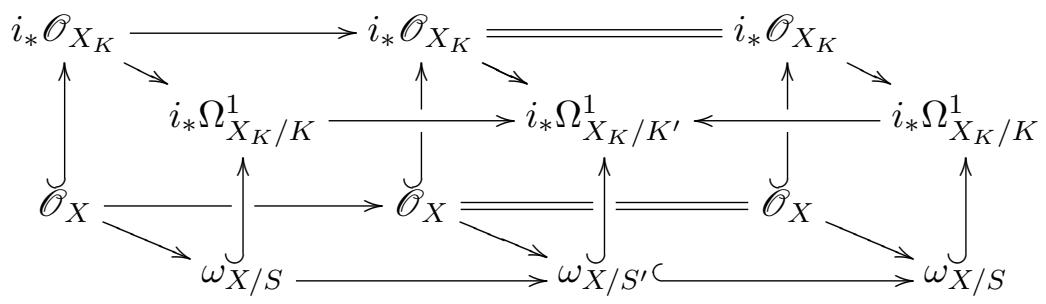

Indeed, the commutativity of the left cube is clear, as all the horizontal arrows are (essentially) multiplication by $\delta$. As for the commutativity of the right cube, it suffices to show that the $\left(\mathscr{O}_{S^{\prime-}}\right.$ linear) derivation

$$
\mathscr{O}_{X} \stackrel{d_{S^{\prime}}}{\longrightarrow} \omega_{X / S^{\prime}} \longrightarrow \omega_{X / S}
$$

coincides with $d_{S}$. Thanks to the flatness of $\omega_{X / S}$ and $\omega_{X / S^{\prime}}$, this may be checked on generic fibers, where it follows from the fact that the canonical isomorphism $\Omega_{X_{K} / K}^{1} \rightarrow \Omega_{X_{K} / K^{\prime}}^{1}$ carries $d_{K}$ to $d_{K^{\prime}}$. Taking hypercohomology of (5.16), we deduce the following commutative diagram of short exact 
sequences of lattices in $H\left(X_{K} / K\right)$ :

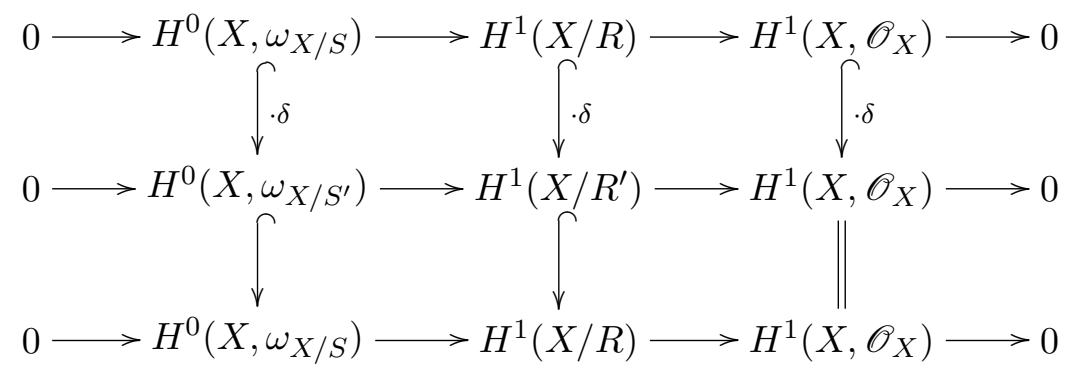

Since $H^{1}\left(X, \omega_{X / S}\right)$ is torsion free, we have

$$
H^{0}\left(X, \omega_{X / S^{\prime}}\right) \simeq H^{0}\left(X, \operatorname{im}\left(\cdot \delta: \omega_{X / S} \rightarrow \omega_{X / S}\right)\right) \simeq \operatorname{im}\left(\cdot \delta: H^{0}\left(X, \omega_{X / S}\right) \rightarrow H^{0}\left(X, \omega_{X / S}\right)\right)
$$

as lattices in $H^{0}\left(X, \Omega_{X_{K} / K}^{1}\right)$, so the top left (vertical) arrow in (5.17) is an isomorphism. It then follows at once from (5.17) that the natural maps (5.13) are isomorphisms.

In general, the integral structure $H(X / R)$ on the Hodge filtration exact sequence $H\left(X_{K} / K\right)$ of the generic fiber may depend on the choice of proper and cohomologically flat model $X$ of $X_{K}$. Remarkably, when $X_{K}$ has an admissible model, the integral structure $H(X / R)$ for admissible models $X$ is intrinsic to $X_{K}$ : it does not depend on the choice of admissible model $X$. It is moreover functorial in $K$-morphisms of $X_{K}$, and so truly deserves to be called a canonical integral structure.

Theorem 5.11. Keep the notation introduced immediately prior to Proposition 5.8, and suppose that $X_{K}$ is a proper smooth curve over $K$ with an admissible model over $R$.

(1) For any two admissible models $X$ and $X^{\prime}$ of $X_{K}$, there is a unique isomorphism of short exact sequences of finite free $R$-modules

$$
H(X / R) \simeq H\left(X^{\prime} / R\right)
$$

respecting the $K$-fiber identifications.

(2) For any finite and generically étale morphism $\rho: X_{K} \rightarrow Y_{K}$ of proper smooth curves over $K$ that admit admissible models $X$ and $Y$, there are canonical $R$-linear homomorphisms of integral structures

$$
H(Y / R) \stackrel{\rho^{*}}{\longrightarrow} H(X / R) \quad \text { and } \quad H(X / R) \stackrel{\rho_{*}}{\longrightarrow} H(Y / R)
$$

which recover the natural $K$-linear pullback and trace maps induced by $\rho_{K}$ on the Hodge filtration exact sequences $H\left(X_{K} / K\right)$ and $H\left(Y_{K} / K\right)$ after extending scalars to $K$.

(3) The morphisms $\rho^{*}$ and $\rho_{*}$ in (2) are adjoint with respect to the pairing furnished by (5.7). More precisely, under the identifications of $H(X / R)$ and $H(Y / R)$ with their $R$-linear duals as in Proposition 5.8, the morphisms

$$
H(X / R)^{\vee} \stackrel{\left(\rho^{*}\right)^{\vee}}{\longrightarrow} H(Y / R)^{\vee} \quad \text { and } \quad H(X / R) \stackrel{\rho_{*}}{\longrightarrow} H(Y / R)
$$

coincide, as do

$$
H(Y / R)^{\vee} \stackrel{\left(\rho_{*}\right)^{\vee}}{\longrightarrow} H(X / R)^{\vee} \quad \text { and } \quad H(Y / R) \stackrel{\rho^{*}}{\longrightarrow} H(X / R) .
$$


Proof. (1). Uniqueness is obvious, by $R$-freeness. Suppose $X$ and $X^{\prime}$ are admissible models of $X_{K}$. Since any two such models can be dominated by a third thanks to Lemma 3.1, we may suppose there is a (necessarily unique) proper birational map $\rho: X^{\prime} \rightarrow X$ inducing the identity on $K$-fibers. Since $\rho$ is birational and $X$ is normal, the natural map $\mathscr{O}_{X} \rightarrow \rho_{*} \mathscr{O}_{X^{\prime}}$ is an isomorphism. Moreover, Proposition 4.6 shows that the trace map on relative dualizing complexes $\rho_{*} \omega_{X^{\prime} / S} \rightarrow \omega_{X / S}$ is an isomorphism, so we obtain a diagram

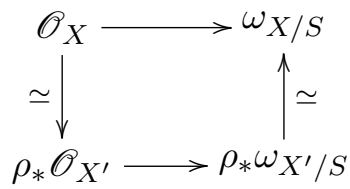

which commutes because this may be checked over $K$, where it is the canonical isomorphism induced by the identification $X_{K} \simeq X_{K}^{\prime}$. We thus obtain a map of complexes

$$
\omega_{X / S}^{\bullet} \stackrel{\simeq}{\longrightarrow} \rho_{*} \omega_{X^{\prime} / S}^{\bullet} \longrightarrow \mathbf{R} \rho_{*} \omega_{X^{\prime} / S}^{\bullet}
$$

that respects the canonical filtrations on both sides, and hence (upon applying $\mathbf{R} \Gamma(X, \cdot)$ ) gives a commutative diagram

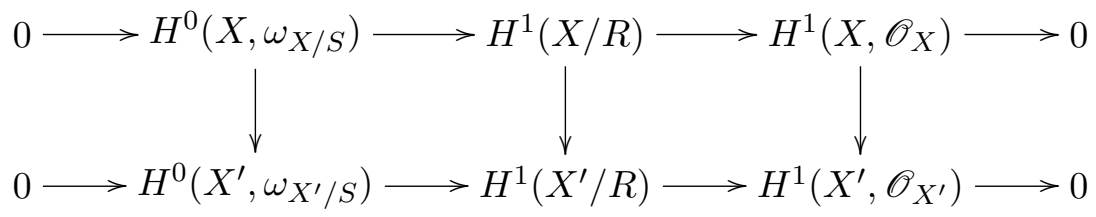

recovering the identification of the Hodge filtrations of $H_{\mathrm{dR}}^{1}\left(X_{K} / K\right)$ and $H_{\mathrm{dR}}^{1}\left(X_{K}^{\prime} / K\right)$ after extending scalars to $K$.

By Proposition 2.6, the right vertical map of (5.18) is an isomorphism. By definition, the left vertical map is the inverse of the map on global sections over $X$ induced from the isomorphism $\rho_{*} \omega_{X^{\prime} / S} \rightarrow \omega_{X / S}$ of Proposition 4.6, and is hence an isomorphism too. It follows that all three vertical maps in (5.18) are isomorphisms.

(2). Let $\rho: X_{K} \rightarrow Y_{K}$ be a finite and generically étale morphism of proper smooth curves over $K$ that have admissible models $X$ and $Y$ over $S$. By Theorem 3.4, there exist admissible models $X^{\prime}$ of $X_{K}$ and $Y^{\prime}$ of $Y_{K}$ that dominate $X$ and $Y$, respectively, and a finite (necessarily generically étale) morphism $\rho^{\prime}: X^{\prime} \rightarrow Y^{\prime}$ recovering $\rho$ on generic fibers. By Proposition 5.7, there exist $R$-linear morphisms of short exact sequences

$$
H\left(Y^{\prime} / R\right) \stackrel{\left(\rho^{\prime}\right)^{*}}{\longrightarrow} H\left(X^{\prime} / R\right) \quad \text { and } \quad H\left(Y^{\prime} / R\right) \stackrel{\left(\rho^{\prime}\right)_{*}}{\longleftarrow} H\left(X^{\prime} / R\right)
$$

recovering the canonical pullback and trace maps, respectively, after extending scalars to $K$. Since $Y^{\prime}$ dominates $Y$ and $X^{\prime}$ dominates $X$, part (1) provides natural isomorphisms of short exact seqences

$$
H\left(Y^{\prime} / R\right) \simeq H(Y / R) \text { and } H\left(X^{\prime} / R\right) \simeq H(X / R)
$$

in a manner recovering the canonical isomorphisms induced by the $K$-fiber identifications $Y_{K}^{\prime} \simeq Y_{K}$ and $X_{K}^{\prime} \simeq X_{K}$ after extending scalars to $K$. We thus obtain the desired maps of integral structures.

(3). The claimed duality of $\rho_{*}$ and $\rho^{*}$ is a straightforward consequence of Proposition 5.5 and basic compatibilities in Grothendieck duality, as we explain below. Denote by $g: Y \rightarrow S$ and $f: X \rightarrow S$ 
the structural morphisms, and for ease of notation, write

$$
(\cdot)_{X}^{\vee}, \quad(\cdot)_{Y}^{\vee} \quad \text { and } \quad(\cdot)_{S}^{\vee}
$$

for the functors

$$
\mathbf{R} \mathscr{H} o m_{X}\left(\cdot, \omega_{f}[-1]\right), \quad \mathbf{R} \mathscr{H} o m_{Y}\left(\cdot, \omega_{g}[-1]\right) \quad \text { and } \quad \mathbf{R} \mathscr{H} o m_{S}\left(\cdot, \mathscr{O}_{S}\right)
$$

respectively. Moreover, if $\bullet$ stands for any one of $g, f, \rho$, we will denote by GD• the Grothendieck duality isomorphism (4.4) attached to $\bullet$. Letting $\rho_{*}$ and $\rho^{*}$ be the pullback and trace morphisms as in (5.5), we claim that there is a commutative diagram of natural quasi-isomorphisms, compatible with filtrations,

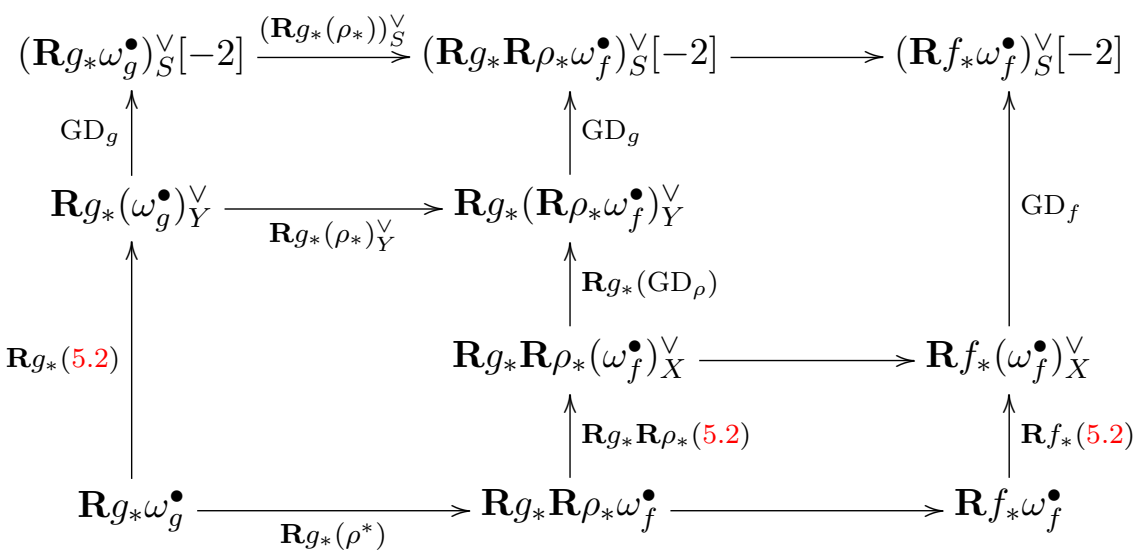

Here, the horizontal maps in the right rectangle are induced by the canonical isomorphism $\mathbf{R} g_{*} \mathbf{R} \rho_{*} \simeq$ $\mathbf{R} f_{*}$ attached to the composite $f=g \circ \rho$. The commutativity of the lower left rectangle follows from Proposition 5.5, by applying $\mathbf{R} g_{*}$. The commutativity of the upper left rectangle is due to the functoriality of Grothendieck's duality isomorphism $\mathrm{GD}_{g}$. The lower right rectangle commutes because the isomorphism of functors $\mathbf{R} g_{*} \mathbf{R} \rho_{*} \simeq \mathbf{R} f_{*}$ is natural. Finally, the upper right rectangle commutes because Grothendieck duality is compatible with composition (see [9, §3.4], especially Lemma 3.4.3).

Applying $H^{1}$ to the large rectangle formed by the outside edges (and using the proof of Proposition 5.8) we deduce the commutativity of the diagram

$$
\begin{aligned}
& H^{1}(Y / R)^{\vee} \stackrel{\left(\rho_{*}\right)^{\vee}}{\longrightarrow} H^{1}(X / R)^{\vee} \\
& (5.7) \uparrow \simeq \quad \simeq \uparrow(5.7) \\
& H^{1}(Y / R) \underset{\rho^{*}}{\longrightarrow} H^{1}(X / R)
\end{aligned}
$$

compatibly with filtrations, as claimed.

\section{Comparison With integral de Rham cohomology}

Fix a proper relative curve $f: X \rightarrow S$. Besides the complex $\omega_{X / S}^{\bullet}$, there is another complex of differentials that provides a lattice in the $K$-vector space $H_{\mathrm{dR}}^{1}\left(X_{K} / K\right)$ : the 2-term "truncated de Rham complex" (in degrees 0,1)

$$
\Omega_{X / S, \leq 1}^{\bullet}:=\mathscr{O}_{X} \stackrel{d}{\longrightarrow} \Omega_{X / S}^{1} .
$$


When $X$ is $S$-smooth, (6.1) and $\omega_{X / S}^{\bullet}$ are canonically ismorphic. In general, thanks to Proposition 5.1 , one has a map of complexes

$$
\Omega_{X / S, \leq 1}^{\bullet} \stackrel{\rho}{\longrightarrow} \omega_{X / S}^{\bullet},
$$

and the discrepancy between the (hyper) cohomologies of these complexes encodes important arithmetic information about $X$ and $X_{K}$.

Remark 6.1. The reader may wonder why we do not also consider the relative de Rham cohomology of $X$ over $S$, given by the hypercohomology of the 3-term de Rham complex

$$
\Omega_{X / S}^{\bullet}:=\mathscr{O}_{X} \stackrel{d}{\longrightarrow} \Omega_{X / S}^{1} \stackrel{d}{\longrightarrow} \Omega_{X / S}^{2} .
$$

Note that $\Omega_{X / S}^{2}$ is $R$-torsion, and is supported only on the non-smooth locus of $f$; in particular, when $X$ is $S$-smooth we have $\Omega_{X / S}^{2}=0$. Simply put, we do not have much to say about the relative de Rham cohomology, as in general it can be somewhat pathological (due to torsion, non-degeneration of the Hodge to de Rham spectral sequence, and the presence of $\Omega_{X / S}^{2}$ which "morally" should be zero as $X$ has relative dimension one over $S$ ). For what little we can prove, see Proposition 6.10 and Remark 6.11 .

In order to apply certain results of Bloch [5] and Liu-Saito [27] in this section, we will need to assume that the residue field $k$ of $R$ is perfect, and that $X$ is regular with geometrically connected generic fiber. Note that although Bloch [5] works only with $R$ equal to the localization at some finite place of the ring of integers in a number field, the proofs of his results that we use below hold for general $R$ with perfect residue field. If the assumption that $k$ is perfect can be removed ${ }^{4}$ in [5] and [27], then our results in this section that depend on these references (i.e. Lemmas 6.4-6.5, Theorem 6.7, and Proposition 6.10) will hold without any hypotheses on $k$ (though we do use the assumption that $X_{K}$ is geometrically connected in the proof of Lemma 6.4 and elsewhere). We will also often need to impose the condition that the greatest common divisor of the multiplicities of the irreducible components of the closed fiber $X_{k}$ of $X$ is 1 ; we will abbreviate this condition by saying that $X_{k}$ is not a multiple fiber. By perfectness of $k$, this is equivalent to requiring that the greatest common divisor of the geometric multiplicities is 1 . Note that under these hypotheses $X$ is admissible (by Proposition $2.7)$.

For a prime $\ell$ different from the residue characteristic of $R$, we recall that the (geometric) Artin conductor of $X$ over $S$ is the positive integer

$$
\operatorname{Art}(X / S):=-\chi\left(X_{\bar{K}}\right)+\chi\left(X_{\bar{k}}\right)+\operatorname{Sw} H_{\text {ét }}^{1}\left(X_{\bar{K}}, \mathbf{Q}_{\ell}\right)
$$

where $\chi$ is the usual étale Euler characteristic and $\mathrm{Sw}$ is the Swan conductor of the $\ell$-adic $\operatorname{Gal}(\bar{K} / K)$ representation $H_{\text {ét }}^{1}\left(X_{\bar{K}}, \mathbf{Q}_{\ell}\right)$. We can also define the (arithmetic) Artin conductor of $X_{K}$ over $K$

$$
\operatorname{Art}\left(X_{K} / K\right):=\operatorname{dim}_{K} H_{\text {ét }}^{1}\left(X_{\bar{K}}, \mathbf{Q}_{\ell}\right)-\operatorname{dim}_{K} H_{\text {ét }}^{1}\left(X_{\bar{K}}, \mathbf{Q}_{\ell}\right)^{I}+\operatorname{Sw}_{\text {ét }}^{1}\left(X_{\bar{K}}, \mathbf{Q}_{\ell}\right) \text {, }
$$

where the vector space in the second term is the subspace of $H_{\text {ét }}^{1}\left(X_{\bar{K}}, \mathbf{Q}_{\ell}\right)$ fixed by an inertia group $I$ of $\operatorname{Gal}(\bar{K} / K)$ (i.e.Art $\left(X_{K} / K\right)$ is the usual Artin conductor of the local $\ell$-adic Galois representation on

\footnotetext{
${ }^{4}$ The referee has pointed out to us that the main difficulty with imperfect $k$ in [5] and [27] is with the definition of the Artin conductor
} 
étale cohomology). Both $\operatorname{Art}(X / S)$ and $\operatorname{Art}\left(X_{K} / K\right)$ are independent of the chosen prime $\ell \neq \operatorname{char}(k)$, and one has the relation ${ }^{5}[5$, Lemma (1.2) (i)]

$$
\operatorname{Art}\left(X_{K} / K\right)=\operatorname{Art}(X / S)-(n-1)
$$

where the closed fiber of $X$ has $n$ geometric irreducible components. Furthermore, if $J_{K}$ is the Jacobian of $X_{K}$, then $\operatorname{Art}\left(X_{K} / K\right)$ is the conductor of $J_{K}[23, \S 1.1]$.

Consider the map of complexes $\rho: \Omega_{X / S, \leq 1}^{\bullet} \rightarrow \omega_{X / S}^{\bullet}$ as in (6.2). Since $\rho$ is an isomorphism on $K$-fibers, the hypercohomology of the mapping cone complex cone $\bullet^{\bullet}(\rho)$ is $R$-torsion, so its Euler characteristic

$$
\chi\left(\operatorname{cone}^{\bullet}(\rho)\right):=\sum_{i \in \mathbf{Z}}(-1)^{i} \operatorname{length}_{R} \mathbf{H}^{i}\left(X, \text { cone }^{\bullet}(\rho)\right)
$$

is defined. A remarkable theorem of $\operatorname{Bloch}^{6}[5, \S 2]$ relates $\chi\left(\operatorname{cone} \bullet^{\bullet}(\rho)\right)$ and $\operatorname{Art}(X / S)$ :

Theorem 6.2 (Bloch). Let $X$ be any regular admissible curve. Then $\operatorname{Art}(X / S)=-\chi\left(\operatorname{cone}^{\bullet}(\rho)\right)$.

Let us denote by

$$
\gamma: H^{0}\left(X, \Omega_{X / S}^{1}\right) \longrightarrow H^{0}\left(X, \omega_{X / S}\right)
$$

the map on cohomology induced by $\rho$ in degree 1 (i.e., the map $H^{0}\left(c_{X / S}\right)$, with $c_{X / S}$ as in Proposition 5.1). In [27], Liu and Saito study the efficient conductor of $X_{K}$ :

$$
\operatorname{Effcond}\left(X_{K} / K\right):=\operatorname{length}_{R} \operatorname{coker}(\gamma) \text {. }
$$

They show [27, Lemma 4] that $\operatorname{Effcond}\left(X_{K} / K\right)$ is independent of the choice of regular proper model $X$ of $X_{K}$, and (using Theorem 6.2) that when the closed fiber of $X$ is not a multiple fiber one has [27, Corollary 2]

$$
\operatorname{Effcond}\left(X_{K} / K\right)=\operatorname{Art}(X / S)-\operatorname{length}_{R} H^{1}\left(X, \Omega_{X / S}^{1}\right) \text { tors. }
$$

From this, they deduce their main theorem [27, Theorem 1]:

Theorem 6.3 (Liu-Saito). Let $X$ be a regular proper $S$-curve with $X_{k}$ not a multiple fiber. Then

$$
\operatorname{Effcond}\left(X_{K} / K\right) \leq \operatorname{Art}\left(X_{K} / K\right) \text {. }
$$

Furthermore, if $X$ is semistable then this inequality is an equality.

We wish to generalize these results to include information about the hypercohomology of the complexes $\Omega_{X / S, \leq 1}^{\bullet}$ and $\omega_{X / S}^{\bullet}$. In order to do this, we first need two lemmas:

Lemma 6.4. Let $X$ be a regular proper and cohomologically flat (i.e. admissible) relative curve over $S$ with geometrically connected generic fiber. Then the natural map

$$
\mathbf{H}^{0}\left(X, \Omega_{X / S, \leq 1}^{\bullet}\right) \longrightarrow H^{0}(X / R)
$$

\footnotetext{
${ }^{5}$ Strictly speaking, [5, Lemma (1.2) (i)] is proved under the assumption that $k$ is finite and $K$ is a number field. When $X_{k}$ is not a multiple fiber, one can use [23, Proposition 1] instead, which does not impose these hypotheses on $k$ or $K$. In fact, we will only need (6.3) when $X_{k}$ is not a multiple fiber.

${ }^{6}$ Our sign convention for $\operatorname{Art}(X / S)$ is the opposite of Bloch's, but is consistent with that of Liu-Saito [27]. As we noted above, although Bloch assumes that $R$ is the localization at some finite place of the ring of integers in a number field, his proof is valid for general $R$ with perfect residue field.
} 
is an isomorphism, and the map $\gamma$ of (6.4) fits into a natural map of short exact sequences

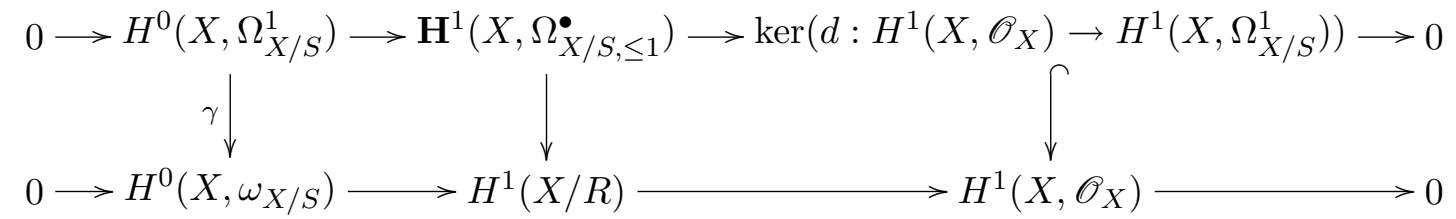

Furthermore, this diagram depends only on the generic fiber $X_{K}$ of $X$ in the following sense: if $X^{\prime}$ is any other choice of regular proper (automatically cohomologically flat by Remark 3.2) model of $X_{K}$, then the diagrams (6.7) corresponding to $X$ and to $X^{\prime}$ are canonically identified.

Proof. Since $X$ is proper with geometrically connected generic fiber, we have $f_{*} \mathscr{O}_{X} \simeq \mathscr{O}_{S}$ so the map $d: H^{0}\left(X, \mathscr{O}_{X}\right) \rightarrow H^{0}\left(X, \Omega_{X / S}^{1}\right)$ is zero. As $\rho$ is compatible with filtrations, we thus have a commutative diagram

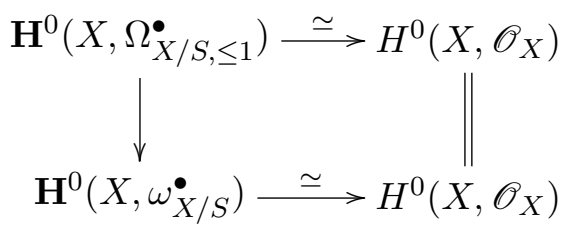

giving the first claim. Moreover, $\rho$ is also compatible with the usual hypercohomology spectral sequences, so using the first claim we obtain the diagram (6.7), in which the right vertical map is obviously injective. To prove the claimed independence of proper regular model, it suffices in view of Theorem 5.11 and the naturality of the formation of (6.7) to show that the top row of (6.7) depends only on $X_{K}$. By the structure theory for regular proper models of $X_{K}$, we may suppose that there is a proper birational map $X^{\prime} \rightarrow X$ extending the identity on $K$-fibers, and we wish to show that the resulting homomorphism

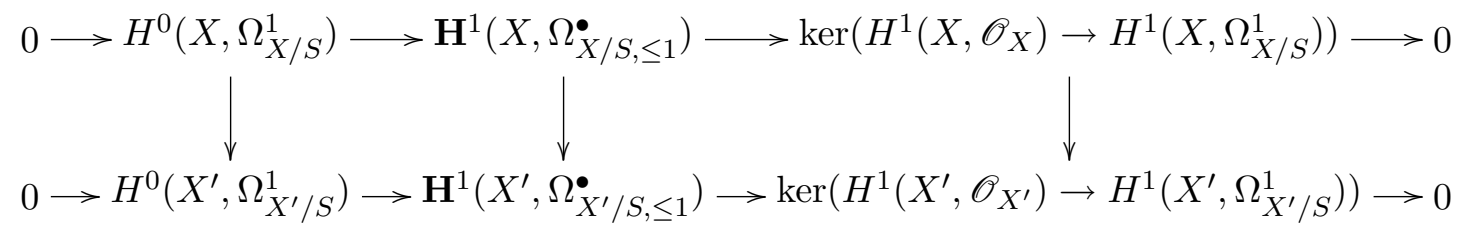

is an isomorphism. The left vertical map is an isomorphism by [27, Lemma 4]. The right vertical map of (6.8) is deduced from the commutative square

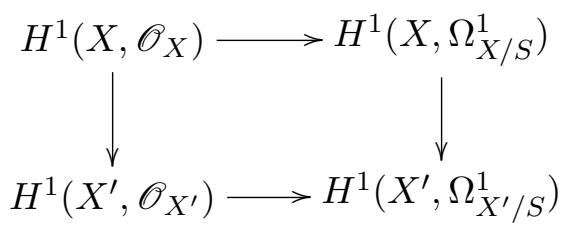

whose left edge is an isomorphism (as $X$ is regular and $X^{\prime} \rightarrow X$ is proper birational) and whose right edge is injective by [27, Remark 3]. We conclude that the right vertical map of (6.8) must be an isomorphism, and hence that the middle vertical map in (6.8) is too.

When the closed fiber of $X$ is not a multiple fiber, then each term in the top row of (6.7) is in fact a submodule of the corresponding term in the bottom row: 
Lemma 6.5. Keep the notation and hypotheses of Lemma 6.4, and suppose in addition that $X_{k}$ is not a multiple fiber. Then all three vertical maps in (6.7) are injective, and the canonical map

$$
\mathbf{H}^{2}\left(X, \Omega_{X / S, \leq 1}^{\bullet}\right) \longrightarrow H^{2}(X / R)
$$

is surjective with kernel $\mathbf{H}^{2}\left(X, \Omega_{X / S, \leq 1}^{\bullet}\right)_{\text {tors }}$.

Proof. When $X_{k}$ is not a multiple fiber, the map $\gamma$ is injective thanks to [27, Corollary 1], so since the right vertical map of (6.7) is always injective, the middle vertical map of (6.7) must be injective as well. For the second claim, as in the proof of Lemma 6.4 we have a commutative diagram

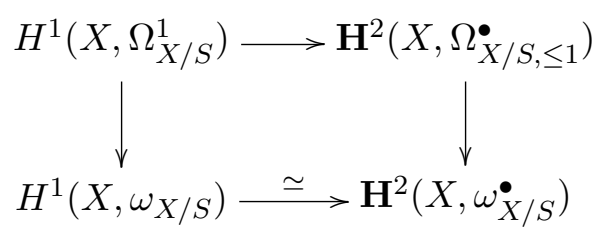

in which the bottom horizontal map is an isomorphism by Proposition 5.7. The left vertical map of this diagram is surjective by [27, Corollary 1] (again, using the assumption that $X_{k}$ is not a multiple fiber), so the same is true of the right vertical map. We know that the right vertical map is moreover an isomorphism after extending scalars to $K$, and that $\mathbf{H}^{2}\left(X, \omega_{X / S}^{\bullet}\right)$ is torsion free thanks to Proposition 5.7 and Theorem 4.4; our second assertion follows immediately.

Definition 6.6. Let $X / S$ be a regular proper and cohomologically flat relative curve with geometrically connected generic fiber. We define the de Rham conductor of $X_{K} / K$ to be the integer

$$
\operatorname{dRcond}\left(X_{K} / K\right):=\operatorname{length}_{R} \operatorname{coker}\left(\mathbf{H}^{1}\left(X, \Omega_{X / S, \leq 1}^{\bullet}\right) \rightarrow \mathbf{H}^{1}\left(X, \omega_{X / S}^{\bullet}\right)\right) .
$$

By Lemma 6.4, this integer depends only on $X_{K}$.

The numerical invariant $\mathrm{dRcond}\left(X_{K} / K\right)$ satisfies the following analogue of (6.6) and Theorem 6.3:

Theorem 6.7. Let $X$ be a regular proper curve over $S$ with geometrically connected generic fiber, and suppose that the closed fiber $X_{k}$ is not a multiple fiber. Then

$$
\operatorname{dRcond}\left(X_{K} / K\right)=\operatorname{Art}(X / S)-\operatorname{length}_{R} \mathbf{H}^{2}\left(X, \Omega_{X, \leq 1}^{\bullet}\right)_{\text {tors }}
$$

Furthermore, we have inequalities

$$
\operatorname{Effcond}\left(X_{K} / K\right) \leq \mathrm{dRcond}\left(X_{K} / K\right) \leq \operatorname{Art}\left(X_{K} / K\right),
$$

and when $X$ is semistable, each of these inequalities is an equality.

Proof. First note that the final assertion concerning the case of semistable $X$ follows immediately from Theorem 6.3, given the inequalities (6.10). Thus, we need only establish (6.9) and (6.10).

As the hypotheses and conclusions are unaffected by base change to the strict henselization of $R$, we may assume that $k$ is separably closed, and hence algebraically closed as $k$ is perfect. For convenience, we will abbreviate $\Omega_{X / S, \leq 1}^{\bullet}$ and $\omega_{X / S}^{\bullet}$ simply by $\Omega^{\bullet}$ and $\omega^{\bullet}$, respectively. The map $\rho: \Omega^{\bullet} \rightarrow \omega^{\bullet}$ fits into an exact triangle

$$
\Omega^{\bullet} \stackrel{\rho}{\longrightarrow} \omega^{\bullet} \longrightarrow \operatorname{cone}^{\bullet}(\rho),
$$


from which we deduce the usual long exact sequence in hypercohomology. Using Lemmas 6.4 and 6.5, we extract from this the exact sequence

$$
0 \longrightarrow \mathbf{H}^{1}\left(\Omega^{\bullet}\right) \longrightarrow \mathbf{H}^{1}\left(\omega^{\bullet}\right) \longrightarrow \mathbf{H}^{1}\left(\operatorname{cone} e^{\bullet}(\rho)\right) \longrightarrow \mathbf{H}^{2}\left(\Omega^{\bullet}\right) \stackrel{\mathbf{H}^{2}(\rho)}{\longrightarrow} \mathbf{H}^{2}\left(\omega^{\bullet}\right) \longrightarrow 0
$$

(with self-evident abbreviated notation), and that one has $\mathbf{H}^{i}\left(\operatorname{cone}^{\bullet}(\rho)\right)=0$ for $i \neq 1$. In particular, we have $\chi\left(\operatorname{cone}^{\bullet}(\rho)\right)=-$ length $_{R} \mathbf{H}^{1}\left(\operatorname{cone}^{\bullet}(\rho)\right)$, so the exact sequence (6.11) tells us that

$$
\operatorname{dRcond}\left(X_{K} / K\right)=-\chi\left(\operatorname{cone}^{\bullet}(\rho)\right)-\operatorname{length}_{R} \operatorname{ker}\left(\mathbf{H}^{2}(\rho)\right)
$$

Applying Theorem 6.2 and using Lemma 6.5 again, we deduce (6.9).

Now the first inequality in (6.10) easily follows from the kernel-cokernel exact sequence arising from the diagram (6.7) and the definition of the efficient and de Rham conductors. In order to establish the second inequality, we proceed as follows. Suppose that the irreducible components of the closed fiber $X_{k}$ are $C_{1}, C_{2}, \ldots, C_{n}$. Thanks to (6.3), to prove the second inequality it suffices to show that length $_{R} \mathbf{H}^{2}\left(\Omega^{\bullet}\right)_{\text {tors }} \geq n-1$. We do this by refining the argument in the proof of [27, Theorem 1 ]. Observe that

$$
\text { length }_{R} \mathbf{H}^{2}\left(X, \Omega^{\bullet}\right)_{\text {tors }} \geq \operatorname{dim}_{k} \mathbf{H}^{2}\left(X, \Omega^{\bullet}\right)_{\text {tors }} \otimes_{R} k=\operatorname{dim}_{k}\left(\mathbf{H}^{2}\left(X, \Omega^{\bullet}\right) \otimes_{R} k\right)-1,
$$

where the equality of the second and third terms results from the fact that the $K$-vector space $\mathbf{H}^{2}\left(X, \Omega_{X / S, \leq 1}^{\bullet}\right) \otimes K$ is one-dimensional. It is not hard to see that the functor $\mathbf{H}^{2}(X, \cdot)$ is right exact on two-term complexes of coherent sheaves, so the the natural base change map

$$
\mathbf{H}^{2}\left(X, \Omega^{\bullet}\right) \otimes_{R} k \longrightarrow \mathbf{H}^{2}\left(X_{k}, \Omega_{X_{k}}^{\bullet}\right)
$$

is surjective. ${ }^{7}$ Thus, we will be done if we can show that $\operatorname{dim}_{k} \mathbf{H}^{2}\left(X_{k}, \Omega_{X_{k}}\right) \geq n$. Give $C_{i}$ its reduced structure. Associated to the natural maps $\iota_{i}: C_{i} \rightarrow X_{k}$ is the (filtered) map of two-term filtered complexes

$$
\Omega_{X_{k}}^{\bullet} \rightarrow \bigoplus_{i} \iota_{i *} \Omega_{C_{i} / k}^{\bullet}
$$

which induces a commutative diagram

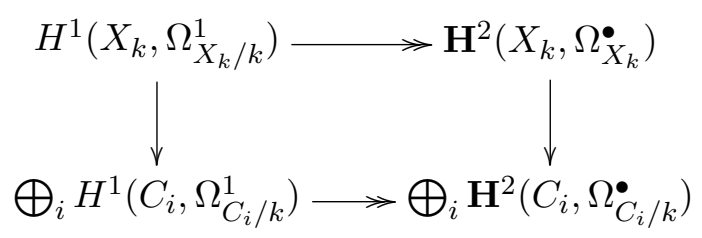

in which the horizontal maps are surjective (due to the vanishing of $H^{2}$ of coherent sheaves on curves over a field). Now the left vertical map is also surjective, since the cokernel of (6.13) is supported on finitely many closed points and $H^{1}$ is right exact and annihilates skyscraper sheaves. It follows that the right vertical map is surjective as well. Provided that each $\mathbf{H}^{2}\left(C_{i}, \Omega_{C_{i} / k}^{\bullet}\right)=\mathbf{H}_{\mathrm{dR}}^{2}\left(C_{i} / k\right)$ is nonzero, we could conclude that $\operatorname{dim}_{k} \mathbf{H}^{2}\left(X_{k}, \Omega_{X_{k}}^{\bullet}\right) \geq n$, so in view of the surjectivity of (6.12) we would be done.

It remains to show that $H_{\mathrm{dR}}^{2}\left(C^{\prime} / k\right) \neq 0$ for any proper $k$-scheme $C^{\prime}$ of dimension 1 . We can adapt the argument using (6.13) to show that $H_{\mathrm{dR}}^{2}\left(C^{\prime} / k\right)$ maps onto $\bigoplus_{j} H_{\mathrm{dR}}^{2}\left(C_{j}^{\prime} / k\right)$, where $\left\{C_{j}^{\prime}\right\}$ are the

\footnotetext{
${ }^{7}$ It is even an isomorphism.
} 
normalizations of the irreducible components of $C^{\prime}$. But the Hodge to de Rham spectral sequence for each smooth curve $C_{j}^{\prime}$ degenerates, so $H_{\mathrm{dR}}^{2}\left(C_{j}^{\prime} / k\right)$ is 1-dimensional over $k$ for each $j$.

Remark 6.8. In fact, we do not need the assumption in Definition 6.6 that $X / S$ be cohomologically flat. Certainly, the map $\rho$ of (6.2) always yields map

$$
\mathbf{H}^{1}\left(X, \Omega_{X / S, \leq 1}^{\bullet}\right) \rightarrow \mathbf{H}^{1}\left(X, \omega_{X / S}^{\bullet}\right)
$$

on hypercohomology, and we can always contemplate the length of the cokernel of this map (even in the absence of the regularity hypothesis). The point is that an analogue of Lemma 6.4 holds when $X$ is only assumed to be regular, and in this way one shows that $\mathrm{dRcond}\left(X_{K} / K\right)$ really only depends on $X_{K}$.

Remark 6.9. Let $X$ be a regular proper curve over $S$ with geometrically connected generic fiber and suppose that the closed fiber $X_{k}$ is not a multiple fiber. It is natural to ask for a conceptual interpretation of the de Rham conductor, relative to the efficient and Artin conductors. In fact, the nonnegative numerical invariants $\mathrm{dRcond}\left(X_{K} / K\right)-\operatorname{Effcond}\left(X_{K} / K\right)$ and $\operatorname{Art}\left(X_{K} / K\right)-\mathrm{dRcond}\left(X_{K} / K\right)$ give a quantitative measure of the degree to which the Hodge to de Rham spectral sequence for the truncated de Rham complex $\Omega_{X / S, \leq 1}^{\bullet}$ degenerates. More precisely, using the construction of (6.7) and Lemma 6.5 , one has a short exact sequence of $R$-modules

$$
0 \longrightarrow \operatorname{im}(d) \longrightarrow H^{1}\left(X, \Omega_{X / S}^{1}\right) \text { tors } \longrightarrow \mathbf{H}^{2}\left(X, \Omega_{X / S, \leq 1}^{\bullet}\right)_{\text {tors }} \longrightarrow 0
$$

where $d$ is the edge map

$$
d: H^{1}\left(X, \mathscr{O}_{X}\right) \longrightarrow H^{1}\left(X, \Omega_{X / S}^{1}\right)
$$

induced by the usual derivation (which must have torsion image due to the degeneration of the Hodge to de Rham spectral sequence of $X_{K}$ over $K$ ). Subtracting (6.6) from (6.9) and using (6.14), we deduce the equality

$$
\mathrm{dRcond}\left(X_{K} / K\right)-\operatorname{Effcond}\left(X_{K} / K\right)=\operatorname{length}_{R}(\operatorname{im}(d)) .
$$

In particular, the de Rham and efficient conductors of $X_{K}$ over $K$ are equal if and only if the Hodge to de Rham spectral sequence for the truncated de Rham complex $\Omega_{X / S, \leq 1}^{\bullet}$ degenerates. In a similar manner, if $n$ denotes the number of geometric irreducible components of $X_{k}$, the proof of Theorem 6.7 and (6.14) show that

$$
\operatorname{Art}\left(X_{K} / K\right)-\operatorname{dRcond}\left(X_{K} / K\right)=\operatorname{length}_{R}\left(H^{1}(X, \Omega)_{\text {tors }} / \operatorname{im}(d)\right)-(n-1),
$$

and this quantity is nonnegative due to (6.10). Thus, the de Rham and Artin conductors are equal if and only if $\operatorname{im}(d)$ is as large as theoretically possible. Note that the right sides of (6.15) and (6.16) are invariants of the generic fiber $X_{K}$, because the left sides are.

We end this section with a proposition that elucidates the structure of the relative de Rham cohomology of $X$ over $S$. As we have indicated, integral de Rham cohomology is often somewhat pathological, so it should come as no surprise that there is little we can say about it in full generality. Nonetheless:

Proposition 6.10. Let $X$ be a proper relative curve over $S$ with geometrically connected generic fiber. Then there are canonical isomorphisms

$$
H_{\mathrm{dR}}^{0}(X / S) \simeq H^{0}\left(X, \mathscr{O}_{X}\right) \quad \text { and } \quad H_{\mathrm{dR}}^{3}(X / S) \simeq \frac{H^{1}\left(X, \Omega_{X / S}^{2}\right)}{d H^{1}\left(X, \Omega_{X / S}^{1}\right)},
$$


as well as an injective map

$$
H_{\mathrm{dR}}^{1}(X / S) \stackrel{\alpha}{\longrightarrow} \mathbf{H}^{1}\left(X, \Omega_{X / S, \leq 1}^{\bullet}\right)
$$

with

$$
\text { length }_{R} \operatorname{coker}(\alpha) \leq \operatorname{length}_{R} H^{0}\left(X, \Omega_{X / S}^{2}\right) .
$$

Moreover, the cokernel of the canonical map $H^{0}\left(X, \Omega_{X / S}^{2}\right) \rightarrow H_{\mathrm{dR}}^{2}(X / S)$ is naturally isomorphic to

$$
\frac{\operatorname{ker}\left(H^{1}\left(X, \Omega_{X / S}^{1}\right) \stackrel{d}{\rightarrow} H^{1}\left(X, \Omega_{X / S}^{2}\right)\right)}{d H^{1}\left(X, \mathscr{O}_{X}\right)} .
$$

In particular, if $X$ is regular and $X_{k}$ is not a multiple fiber then $H_{\mathrm{dR}}^{1}(X / S)$ is torsion-free, and if $X_{k}$ is in addition generically smooth, then $H_{\mathrm{dR}}^{3}(X / S)=0$.

Proof. A straightforward analysis of the Hodge to de Rham spectral sequence yields the claimed identifications of $H_{\mathrm{dR}}^{0}$ and $H_{\mathrm{dR}}^{3}$, as well as the statements concerning $H_{\mathrm{dR}}^{2}$ and $\alpha$. When $X$ is regular and $X_{k}$ is not a multiple fiber, we may apply Lemma 6.5 to deduce that we have an injection $H_{\mathrm{dR}}^{1}(X / S) \hookrightarrow H^{1}(X / R)$; the latter module is torsion free by Proposition 5.7. When $X_{k}$ is generically smooth, $\Omega_{X / S}^{2}$ is a skyscraper sheaf (as in this case the non-smooth locus of $X$ over $S$ consists of a finite number of closed points in the closed fiber), so $H^{1}\left(X, \Omega_{X / S}^{2}\right)=0$.

Remark 6.11. It is natural to ask if one can give a bound for $L:=\operatorname{length} H^{0}\left(X, \Omega_{X / S}^{2}\right)$. Using the results of this section, it is not hard to show that when $X$ is regular and $X_{k}$ is not a multiple fiber, we have $L \geq n-1$, where $X_{k}$ has $n$ geometric irreducible components. When $X_{k}$ is in addition generically smooth, one can also show that $L \leq \operatorname{Art}(X / S)$.

\section{BASE CHANGE}

Let $X_{K}$ be a smooth proper and geometrically connected curve over $K$ with an admissible model $X$ over $R$, and fix a local extension of discrete valuation rings $R \rightarrow R^{\prime}$ with associated fraction field extension $K \rightarrow K^{\prime}$. Extension of scalars gives an integral structure $H^{1}(X / R) \otimes_{R} R^{\prime}$ on $H_{\mathrm{dR}}^{1}\left(X_{K^{\prime}} / K^{\prime}\right)$. When $X_{K^{\prime}}$ has an admissible model $X^{\prime}$ over $R^{\prime}$, it is natural to ask how this lattice relates to the canonical integral structure $H^{1}\left(X^{\prime} / R^{\prime}\right)$. If the residue field extension $k \rightarrow k^{\prime}$ is separable and the relative ramification index $e\left(R^{\prime} / R\right)$ is 1 , then $X^{\prime}=X \times_{R} R^{\prime}$ is an admissible model of $X_{K^{\prime}}$, as we noted in the discussion preceding Proposition 3.5, and hence $H^{1}(X / R) \otimes_{R} R^{\prime}$ and $H^{1}\left(X^{\prime} / R^{\prime}\right)$ are canonically isomorphic. This includes the cases $R^{\prime}=\widehat{R}$ and $R^{\prime}=R^{\text {sh }}$, so the most interesting cases have $R$ complete and $\left[K^{\prime}: K\right]$ finite. For general base change, these two lattices need not coincide, nor must either lattice necessarily contain the other. Nonetheless, we can estimate how far apart these lattices inside $H_{\mathrm{dR}}^{1}\left(X_{K^{\prime}} / K^{\prime}\right)$ are in general:

Theorem 7.1. Let $X_{K}$ be a smooth, proper and geometrically connected curve over $K$ with an admissible model $X$ over $R$, and assume that $\left[K^{\prime}: K\right]$ is finite and that $X_{K^{\prime}}$ has an admissible model $X^{\prime}$ over $R^{\prime}$. Set

$$
L:=H^{1}(X / R) \otimes_{R} R^{\prime} \quad \text { and } \quad L^{\prime}:=H^{1}\left(X^{\prime} / R^{\prime}\right),
$$

considered as $R^{\prime}$-lattices inside $H_{\mathrm{dR}}^{1}\left(X_{K^{\prime}} / K^{\prime}\right)$. Denote by e the relative ramification index of $R^{\prime}$ over $R$, fix a uniformizer $\pi$ of $R$, and put $E:=\operatorname{Effcond}\left(X_{K} / K\right)$ (see (6.5) for the definition). Then

(1) $\pi^{E} L \subseteq L^{\prime}$ and $\pi^{E} L^{\prime} \subseteq L$. 
(2) Each of $L / L \cap L^{\prime}$ and $L^{\prime} / L \cap L^{\prime}$ is of finite $R^{\prime}$-length at most eE.

Proof. As usual, put $S=\operatorname{Spec} R$ and $S^{\prime}=\operatorname{Spec} R^{\prime}$. Thanks to Theorem 5.11 and Proposition 3.5, we may suppose that $X$ is regular and that $X^{\prime}$ is the normalization of $X_{S^{\prime}}:=X \times_{S} S^{\prime}$. The normalization map $\rho: X^{\prime} \rightarrow X_{S^{\prime}}$ is then finite and birational, so we have natural maps $\mathscr{O}_{X_{S^{\prime}}} \rightarrow \rho_{*} \mathscr{O}_{X^{\prime}}$ and $\rho_{*} \omega_{X^{\prime} / S^{\prime}} \rightarrow \omega_{X_{S^{\prime}} / S^{\prime}}$ whose cokernels are supported on the closed fiber of $X_{S^{\prime}}$. These maps fit naturally into diagrams of 2-term (horizontal) complexes on $X_{S^{\prime}}$
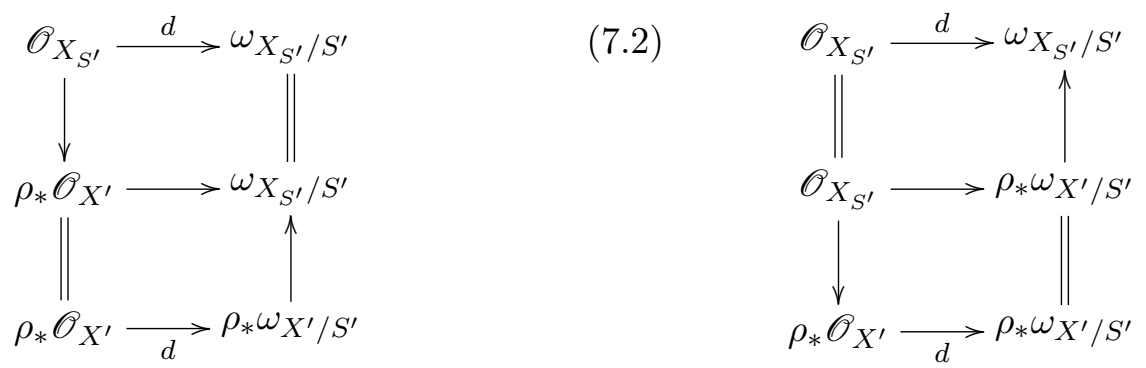

where

in each case the middle horizontal arrow is deduced from the bottom one, and the diagrams commute as this may be checked over the generic fiber, where it is obvious. We claim that for each 2-term (horizontal) complex occurring in (7.1) and (7.2), the usual "Hodge to de Rham" spectral sequence for hypercohomology degenerates at the $E_{1}$-stage. Due to such degeneration on the generic fiber, as in the proof of Proposition 5.7 it suffices to show that $H^{i}\left(X_{S^{\prime}}, \omega_{X_{S^{\prime}} / S^{\prime}}\right)$ and $H^{i}\left(X_{S^{\prime}}, \rho_{*} \omega_{X^{\prime} / S^{\prime}}\right)$ are torsion free for $i=0,1$. This follows easily from Theorem 4.4 (using that $X$ and $X^{\prime}$ are admissible) after making the canonical identifications

$$
H^{i}\left(X_{S^{\prime}}, \omega_{X_{S^{\prime}} / S^{\prime}}\right) \simeq H^{i}\left(X, \omega_{X / S}\right) \otimes_{R} R^{\prime} \text { and } H^{i}\left(X_{S^{\prime}}, \rho_{*} \omega_{X^{\prime} / S^{\prime}}\right) \simeq H^{i}\left(X^{\prime}, \omega_{X^{\prime} / S^{\prime}}\right)
$$

that result from the compatibility of the dualizing sheaf with base change and the finiteness of $\rho$, respectively. We deduce from (7.1) and (7.2) the following commutative diagrams of short exact sequences of free $R^{\prime}$-modules:
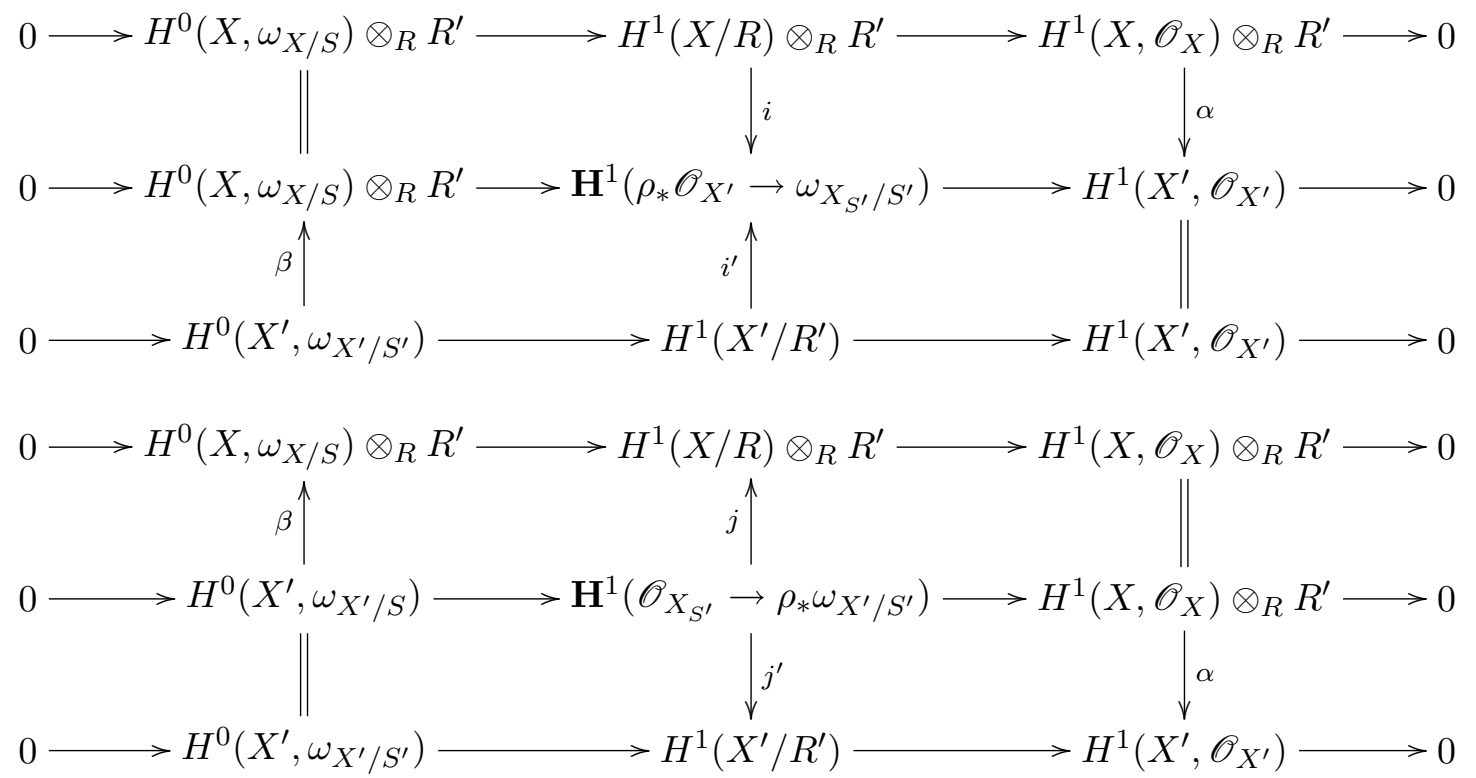
Since $\alpha$ and $\beta$ have torsion-free sources and become isomorphisms after extending scalars to $K^{\prime}$, we conclude that both maps are injective and have finite-length cokernels. The diagrams (7.3) and (7.4) then imply that $i$ and $j$ are injective, and that (via the natural maps)

$$
\text { coker } i \simeq \operatorname{coker} \alpha \simeq \operatorname{coker} j^{\prime} \text { and } \operatorname{coker} i^{\prime} \simeq \operatorname{coker} \beta \simeq \operatorname{coker} j .
$$

On the other hand, Grothendieck duality for $\rho$ ensures that the maps $\alpha$ and $\beta$ are dual, and that there is a canonical isomorphism of finite length $R^{\prime}$-modules

$$
\operatorname{coker} \beta \simeq \operatorname{Ext}_{R^{\prime}}^{1}\left(\operatorname{coker} \alpha, R^{\prime}\right) .
$$

In particular coker $\alpha$ and coker $\beta$ have the same $R^{\prime}$-length. Thus, Theorem 7.1 follows easily from (7.5), the diagrams (7.3) and (7.4), and the bound

$$
\text { length }_{R^{\prime}}(\operatorname{coker} \beta) \leq e E .
$$

To prove (7.6), we first claim that $\beta$ fits into a commutative diagram

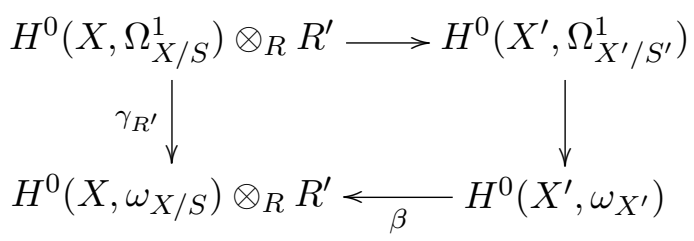

where the vertical maps are induced by the canonical maps from differentials to dualizing sheaves (i.e. the map $\gamma_{R^{\prime}}$ is the base change of (6.4); cf. also Proposition 5.1) and the top horizontal map is the usual pullback map on differentials associated to $\rho: X^{\prime} \rightarrow X_{S^{\prime}}$. Indeed, since $H^{0}\left(X, \omega_{X / S}\right) \otimes_{R} R^{\prime}$ is torsion free, the commutativity of (7.7) may be checked after extending scalars to $K^{\prime}$, where it follows immediately from the construction of the maps involved. As a particular consequence of the commutativity of (7.7), we deduce that

$$
\text { length }_{R^{\prime}}(\operatorname{coker} \beta) \leq \text { length }_{R^{\prime}}\left(\operatorname{coker} \gamma_{R^{\prime}}\right) .
$$

On the other hand, by the definition (6.5) of $E:=\operatorname{Effcond}\left(X_{K} / K\right)$ and the fact that $\gamma_{R^{\prime}}$ is obtained from the map $\gamma$ of (6.4) by base change, we have

$$
\text { length }_{R^{\prime}}\left(\operatorname{coker} \gamma_{R^{\prime}}\right)=e \text { length }_{R}(\operatorname{coker} \gamma)=e E,
$$

and the required bound (7.6) follows at once.

Remarks 7.2. As we observed immediately preceding Proposition 3.5, the hypotheses of Theorem 7.1 are satisfied by any smooth and geometrically connected curve $X_{K}$ equipped with a rational point over a tamely ramified extension of $K$.

Note that the bounds given in Theorem 7.1 (1) do not depend on $R^{\prime}$ at all. We do not know if these bounds are sharp; however, it is easy to see that if one of $L, L^{\prime}$ contains the other, then they must be equal (as lattices inside $H_{\mathrm{dR}}^{1}\left(X_{K^{\prime}} / K^{\prime}\right)$ ). Indeed, suppose, for example, that $L \subseteq L^{\prime}$. Then from either of the diagrams (7.3) or (7.4), we deduce a commutative diagram

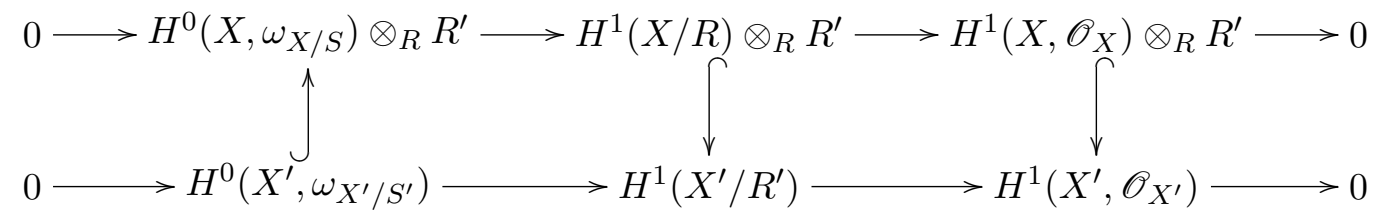


from which it follows (by a simple diagram chase) that the left vertical injection is an isomorphism. By Grothendieck duality for $\rho: X^{\prime} \rightarrow X_{R^{\prime}}$, the right map is also an isomorphism, so the same is true for the middle map. The argument in the case of the reverse inclusion of lattices is similar.

Supposing that $k$ is perfect, when the closed fiber of $X$ is not a multiple fiber (for example, when $X_{K}$ has a rational point over an unramified extension of $K$ ), we may apply Theorem 6.3 of Liu-Saito to bound $E$ above by $\operatorname{Art}\left(X_{K} / K\right)$.

\section{REFERENCES}

[1] Shreeram Abhyankar. Simultaneous resolution for algebraic surfaces. Amer. J. Math., 78:761-790, 1956.

[2] Shreeram Abhyankar. Resolution of singularities of arithmetical surfaces. In Arithmetical Algebraic Geometry (Proc. Conf. Purdue Univ., 1963), pages 111-152. Harper \& Row, New York, 1965.

[3] Michael Artin. Algebraization of formal moduli. I. In Global Analysis (Papers in Honor of K. Kodaira), pages 21-71. Univ. Tokyo Press, Tokyo, 1969.

[4] Michael Artin. Lipman's proof of resolution of singularities for surfaces. In Gary Cornell and Joseph H. Silverman, editors, Arithmetic geometry (Storrs, Conn., 1984), pages 267-287. Springer, New York, 1986. Papers from the conference held at the University of Connecticut, Storrs, Connecticut, July 30-August 10, 1984.

[5] Spencer Bloch. de Rham cohomology and conductors of curves. Duke Math. J., 54(2):295-308, 1987.

[6] Siegfried Bosch, Ulrich Güntzer, and Reinhold Remmert. Non-Archimedean analysis, volume 261 of Grundlehren der Mathematischen Wissenschaften [Fundamental Principles of Mathematical Sciences]. Springer-Verlag, Berlin, 1984. A systematic approach to rigid analytic geometry.

[7] Siegfried Bosch, Werner Lütkebohmert, and Michel Raynaud. Néron models, volume 21 of Ergebnisse der Mathematik und ihrer Grenzgebiete (3) [Results in Mathematics and Related Areas (3)]. Springer-Verlag, Berlin, 1990.

[8] Bryden Cais. Canonical extensions of Néron models of Jacobians. Submitted, 2008.

[9] Brian Conrad. Grothendieck duality and base change, volume 1750 of Lecture Notes in Mathematics. Springer-Verlag, Berlin, 2000.

[10] Brian Conrad. Arithmetic moduli of generalized elliptic curves. J. Inst. Math. Jussieu, 6(2):209-278, 2007.

[11] Brian Conrad, Bas Edixhoven, and William Stein. $J_{1}(p)$ has connected fibers. Doc. Math., 8:331-408 (electronic), 2003.

[12] Pierre Deligne. Théorie de Hodge. II. Inst. Hautes Études Sci. Publ. Math., (40):5-57, 1971.

[13] Pierre Deligne and Luc Illusie. Relèvements modulo $p^{2}$ et décomposition du complexe de de Rham. Invent. Math., 89(2):247-270, 1987.

[14] Pierre Deligne and David Mumford. The irreducibility of the space of curves of given genus. Inst. Hautes Études Sci. Publ. Math., (36):75-109, 1969.

[15] Pierre Deligne and Michael Rapoport. Les schémas de modules de courbes elliptiques. In Modular functions of one variable, II (Proc. Internat. Summer School, Univ. Antwerp, Antwerp, 1972), pages 143-316. Lecture Notes in Math., Vol. 349. Springer, Berlin, 1973.

[16] Renée Elkik. Rationalité des singularités canoniques. Invent. Math., 64(1):1-6, 1981.

[17] Matthew Emerton. On the interpolation of systems of eigenvalues attached to automorphic Hecke eigenforms. Invent. Math., 164(1):1-84, 2006.

[18] Alexander Grothendieck and Jean Dieudonné. Éléments de géométrie algébrique. Inst. Hautes Études Sci. Publ. Math., (4,8,11,17,20,24,28,37), 1960-7.

[19] Robin Hartshorne. Residues and duality. Lecture notes of a seminar on the work of A. Grothendieck, given at Harvard 1963/64. With an appendix by P. Deligne. Lecture Notes in Mathematics, No. 20. Springer-Verlag, Berlin, 1966.

[20] Stephen Lichtenbaum. Curves over discrete valuation rings. Amer. J. Math., 90:380-405, 1968.

[21] Joseph Lipman. Rational singularities, with applications to algebraic surfaces and unique factorization. Inst. Hautes Études Sci. Publ. Math., (36):195-279, 1969.

[22] Joseph Lipman. Desingularization of two-dimensional schemes. Ann. Math. (2), 107(1):151-207, 1978.

[23] Qing Liu. Conducteur et discriminant minimal de courbes de genre 2. Compositio Math., 94(1):51-79, 1994.

[24] Qing Liu. Algebraic geometry and arithmetic curves, volume 6 of Oxford Graduate Texts in Mathematics. Oxford University Press, Oxford, 2002. Translated from the French by Reinie Erné, Oxford Science Publications. 
[25] Qing Liu. Stable reduction of finite covers of curves. Compos. Math., 142(1):101-118, 2006.

[26] Qing Liu and Dino Lorenzini. Models of curves and finite covers. Compositio Math., 118(1):61-102, 1999.

[27] Qing Liu and Takeshi Saito. Inequality for conductor and differentials of a curve over a local field. J. Algebraic Geom., 9(3):409-424, 2000.

[28] Hideyuki Matsumura. Commutative ring theory, volume 8 of Cambridge Studies in Advanced Mathematics. Cambridge University Press, Cambridge, second edition, 1989. Translated from the Japanese by M. Reid.

[29] Barry Mazur and William Messing. Universal extensions and one dimensional crystalline cohomology. SpringerVerlag, Berlin, 1974. Lecture Notes in Mathematics, Vol. 370.

[30] Barry Mazur and Ken Ribet. Two-dimensional representations in the arithmetic of modular curves. Astérisque, (196-197):6, 215-255 (1992), 1991. Courbes modulaires et courbes de Shimura (Orsay, 1987/1988).

[31] Michel Raynaud. Spécialisation du foncteur de Picard. Inst. Hautes Études Sci. Publ. Math., (38):27-76, 1970.

[32] Michel Raynaud and Laurent Gruson. Critères de platitude et de projectivité. Techniques de "platification" d'un module. Invent. Math., 13:1-89, 1971.

[33] Peter Schneider and Jeremy Teitelbaum. Banach space representations and Iwasawa theory. Israel J. Math., 127:359$380,2002$.

Centre de ReCherches mathématiques, Montréal

E-mail address: bcais@math.mcgill.ca 